\title{
Altered keratinocyte differentiation is an early driver of keratin mutation-based palmoplantar keratoderma
}

\author{
Abigail G. Zieman ${ }^{1,2, \dagger}$, Brian G. Poll1,3, Jingqun $\mathrm{Ma}^{2}$ and \\ Pierre A. Coulombe ${ }^{1,2, *}$ \\ ${ }^{1}$ Department of Biochemistry and Molecular Biology, Bloomberg School of Public Health, Johns Hopkins \\ University, Baltimore, MD 21287, USA, ${ }^{2}$ Department of Cell and Developmental Biology, University of Michigan \\ Medical School, Ann Arbor, MI 48109, USA and ${ }^{3}$ Department of Physiology, School of Medicine, Johns Hopkins \\ University, Baltimore, MD 21287, USA
}

*To whom correspondence should be addressed at: Department of Cell and Developmental Biology, University of Michigan Medical School, 3071 Biomedical Sciences Research Building, 109 Zina Pitcher Place, Ann Arbor, MI 48109, USA. Tel: 734-615-7509; Fax: 734-615-8500;

Email: coulombe@umich.edu

\begin{abstract}
The type I intermediate filament keratin 16 (KRT16 gene; K16 protein) is constitutively expressed in ectoderm-derived appendages and in palmar/plantar epidermis and is robustly induced when the epidermis experiences chemical, mechanical or environmental stress. Missense mutations at the KRT16 locus can cause pachyonychia congenita (PC, OMIM:167200) or focal non-epidermolytic palmoplantar keratoderma (FNEPPK, OMIM:613000), which each entail painful calluses on palmar and plantar skin. Krt16-null mice develop footpad lesions that mimic PC-associated PPK, providing an opportunity to decipher its pathophysiology, and develop therapies. We report on insight gained from a genome-wide analysis of gene expression in PPK-like lesions of Krt16-null mice. Comparison of this data set with publicly available microarray data of PPK lesions from individuals with PC revealed significant synergies in gene expression profiles. Keratin 9 (Krt9/K9), the most robustly expressed gene in differentiating volar keratinocytes, is markedly downregulated in Krt16-null paw skin, well-ahead of lesion onset, and is paralleled by pleiotropic defects in terminal differentiation. Effective prevention of PPK-like lesions in Krt16-null paw skin (via topical delivery of the Nrf2 inducer sulforaphane) involves the stimulation of Krt9 expression. These findings highlight a role for defective terminal differentiation and loss of Krt9/K9 expression as additional drivers of PC-associated PPK and highlight restoration of KRT9 expression as a worthy target for therapy. Further, we report on the novel observation that keratin 16 can localize to the nucleus of epithelial cells, implying a potential nuclear function that may be relevant to PC and FNEPPK.
\end{abstract}

\section{Introduction}

Hereditary palmoplantar keratoderma (PPK) results from inherited defects in a broad variety of genes encoding structural proteins, effectors of terminal differentiation, ion channels and other effectors (1). PPK lesions involve dramatic epidermal thickening and hyperkeratosis of palms and soles and can be painful and debilitating for patients. PPK disorders are individually rare but represent a significant clinical problem. The involvement of

† Abigail G. Zieman, http://orcid.org/0000-0001-8236-207X

Received: October 1, 2018. Revised: January 8, 2019. Accepted: March 4, 2019

@ The Author(s) 2019. Published by Oxford University Press. All rights reserved.

For Permissions, please email: journals.permissions@oup.com 
a broad variety of genes in the genetic etiology of PPK suggests that their protein products function in an integrated fashion to promote normal homeostasis of the epidermis in volar skin. A better understanding of the pathophysiology of PPK lesions is needed to better understand normal homeostasis in volar skin and support the development of effective therapeutic strategies for PPK, which are lacking at present.

PPK disorders arising from mutations in keratin genes are either epidermolytic, in that they entail cell fragility and lysis in the differentiating layers of epidermis, or non-epidermolytic, in which there is scant evidence of cell fragility. Mutations in keratin 9 (KRT9, human gene; Krt9, mouse gene; K9, protein) or KRT1 cause diffuse and epidermolytic PPK (EPPK, OMIM: 144200) $(2,3)$, whereas mutations in KRT16 or KRT6c can cause a presentation of focal non-epidermolytic PPK independent of other symptoms associated with the disease pachyonychia congenita (PC; FNEPPK, OMIM: 613000; PPKNEFD, OMIM: 615735) (4,5). Mutations in the wound inducible keratins KRT16, KRT17, KRT6a or KRT6b each can cause PC types 1-4 (PC-1, OMIM: 167200; PC-2, OMIM: 167210; PC-3, OMIM: 615726; PC-4, OMIM: 615728), which involve anomalies in ectoderm-derived epithelial appendages in addition to painful, debilitating and non-epidermolytic PPK (6-9). The pathophysiology of all PPKs including PC-associated PPK is partially understood at present, and only palliative treatments are available for these conditions. There are currently only two mouse models considered to mimic keratin-based PPK disorders. Mice genetically null for Krt16 spontaneously develop footpad lesions mimicking non-epidermolytic PPK typical of PC patients (10-12). Mice genetically null for Krt9 develop footpad lesions that mimic epidermolytic PPK lesions (13).

Krt16-null mice, originally generated in the inbred C57Bl/6 strain background, develop oral lesions shortly after birth and footpad skin lesions as young adults (10). Both features are relevant to PC. The footpad skin lesions entail a dramatic thickening of the entire epidermis, particularly at sites of contact with the substratum, and display gross misregulation of danger-associated molecular patterns (DAMPs or alarmins) and several other barrier homeostasis genes (11). Transfer of the Krt16-null allele to the FVB/N strain background yields a qualitatively similar albeit quantitatively less-severe phenotype (14), likely emulating the known importance of genetic background in PC $(4,15,16)$. Prior to onset of PPK-like lesions, Krt16-null mouse paw skin exhibits oxidative stress secondary to hypoactive Keap1-Nrf2 signaling, which also occurs in PPK lesions of PC patients (17). Topical activation of Nrf2 in the footpad skin of Krt16-null mice, achieved either via sulforaphane (SF) treatment in male mice or co-treatment of SF and diarylpropionitrile (DPN) in female mice, can prevent the genesis of PPK-like lesions in Krt16-null mice $(17,18)$.

Studies of Krt16- and Krt9-null mouse models have so far entailed the use of biased approaches to defining the pathophysiology of PPK-like lesions in footpad skin $(10,11,13,17,18)$. To broaden the scope of our analysis of the mechanisms potentially at play, we assessed global changes in gene expression in Krt16-null footpad skin lesions. This approach validated and significantly extended previous studies showing that skin barrier genes are misregulated in the absence of Krt16 and yielded new insight into a role for $\mathrm{K} 16$ in regulating terminal differentiation of volar epidermis. Comparison of this data set with published microarray data of human cases of PC highlights the heterogeneity and complexity of PPK. Our findings point to impaired terminal differentiation as a key driver of pathophysiology beginning at an early stage of PC-associated PPK and identify restoration of KRT9 expression as a potential novel ther- apeutic strategy of interest for the treatment of PC-associated PPK lesions.

\section{Results}

\section{Genome-wide microarray analysis of gene expression in Krt16-null footpad skin lesions}

To explore the global gene expression changes that occur in Krt16-null paw skin, RNA isolated from the footpad skin (epidermis and dermis) of 2 month old Krt16-null and wild-type (WT) littermate controls was subjected to microarray analysis using the Affymetrix Mouse Transcriptome Array 1.0 plate (data sets are publicly available under GEO accession GSE117375). Differentially expressed genes were ranked by fold change and binned by standard deviations (SDs) from WT gene expression using a normal Gaussian distribution. Using a cutoff of 2 SDs from WT expression (the top 5\% of transcripts detected), there was a total of 5327 differentially expressed transcripts between WT and Krt16-null paw skin including known protein coding genes, microRNAs and predicted genes (2583 upregulated, 2744 downregulated) (Fig. 1A). Of the 2583 upregulated transcripts in Krt16-null paw skin, 700 were binned as $\geq 3$ SD above WT, and, of those, 264 transcripts were binned as $\geq 6$ SD above WT expression. Of the 2744 downregulated transcripts in Krt16-null paw skin, 808 were binned as $\leq 3$ SD below WT, and 256 transcripts were binned as $\leq 6 \mathrm{SD}$ below WT. The top 264 upregulated transcripts and top 256 downregulated transcripts in Krt16-null paw skin were subjected to gene ontology (GO) analysis using PANTHER $(19,20)$. Biological processes involved in inflammation and the immune response are enriched in the upregulated genes in Krt16-null paw skin lesions (Supplementary Material, Table 1), thus confirming and extending previous studies (11). Biological processes involved in muscle function and development are enriched in the downregulated genes in Krt16-null paw skin lesions (Supplementary Material, Table 2), which could reflect the mild growth defect and/or reduced mobility exhibited by Krt16-null mice (10).

This microarray data set was also analyzed using Ingenuity Pathway Analysis (IPA) software to determine potential upstream regulators that could account for differentially expressed genes between genotypes. Of the top 10 regulators predicted to be activated in Krt16-null paw skin, half are cytokines including TNF, CSF2, IL-1 $\beta$, IL-2 and IL$1 \alpha$ (Supplementary Material, Table 3). Additionally, the proinflammatory transcription factor NF-kB and kinase ERK1/2 are also predicted to be activated in Krt16-null paw skin lesions (Supplementary Material, Table 3), again consistent with the established notion that inflammation and dysregulated innate immunity contribute to the pathology of Krt16-null footpad lesions (11).

Four genes (S100A8, S100A9, Stfa1 and Sprr2d) previously found to be dramatically upregulated in Krt16-null skin using target-specific Real Time-quantitative Polymerase Chain Reaction (RT-qPCR), both in the lesions that spontaneously arise in volar paw skin and in phenotypically normal ear skin subjected to mechanical abrasion or chemical irritants (11), are identified among the top 10 most significantly upregulated genes in this analysis (Fig. 1B). Moreover, several additional genes showing significant upregulation in the lesions, including barrier homeostasis genes and DAMPs (Supplementary Material, Table 4), have also been previously shown to be elevated in Krt16-null paw skin lesions using target-specific RT-qPCR assays (11), further attesting to the robustness of the microarray findings. 

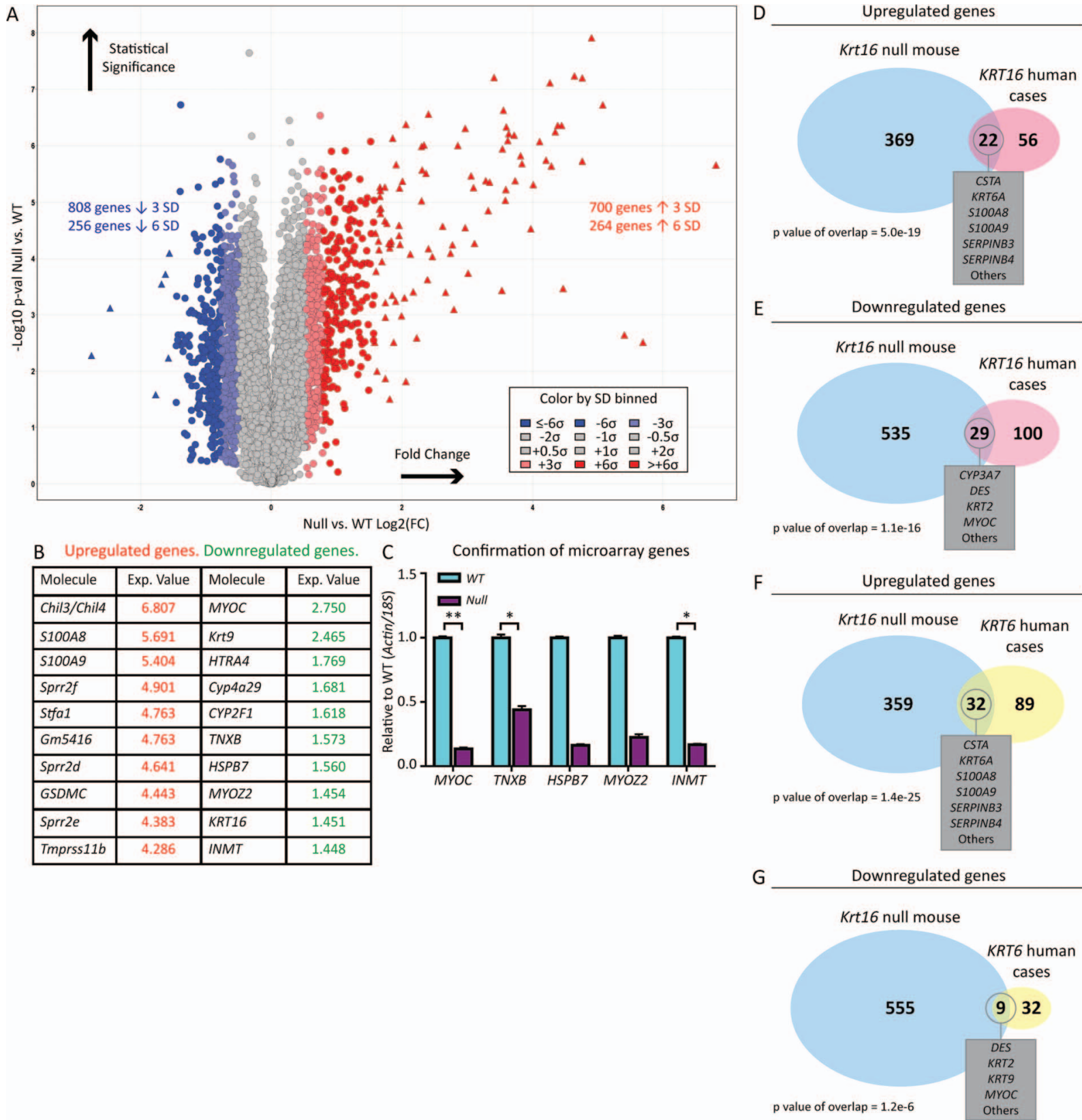

B Upregulated genes. Downregulated genes.
\begin{tabular}{|l|c|l|c|}
\hline Molecule & Exp. Value & Molecule & Exp. Value \\
\hline Chil3/Chil4 & 6.807 & MYOC & 2.750 \\
\hline S100A8 & 5.691 & Krt9 & 2.465 \\
\hline S100A9 & 5.404 & HTRA4 & 1.769 \\
\hline Sprr2f & 4.901 & Cyp4a29 & 1.681 \\
\hline Stfa1 & 4.763 & CYP2F1 & 1.618 \\
\hline Gm5416 & 4.763 & TNXB & 1.573 \\
\hline Sprr2d & 4.641 & HSPB7 & 1.560 \\
\hline GSDMC & 4.443 & MYOZ2 & 1.454 \\
\hline Sprr2e & 4.383 & KRT16 & 1.451 \\
\hline Tmprss11b & 4.286 & INMT & 1.448 \\
\hline
\end{tabular}

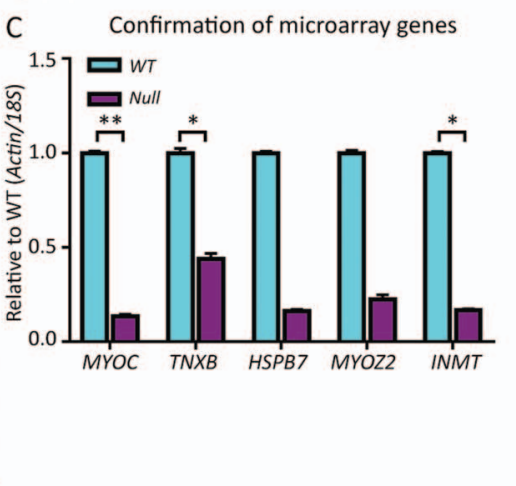

Figure 1. Analysis of global gene expression in Krt16-null footpad lesions and comparison to human PC cases. (A) Volcano plot depicting differentially expressed genes in Krt16-null paw skin lesions relative to WT controls. (B) Top 10 genes differentially regulated in Krt16-null paw skin from established paw lesions compared to WT littermate controls. (C) Validation of downregulated genes identified by microarray analysis by RT-qPCR in Krt16-null paw skin lesions. N = 4 mice/genotype. Error bars are SEM. ${ }^{*} P<0.05,{ }^{* *} P<0.01$. (D) Overlap in the significantly changed genes that are upregulated between Krt16-null paw skin lesions and KRT16 human cases. (E) Overlap in the significantly changed genes that are downregulated between Krt16-null paw skin lesions and KRT16 human cases. (F) Overlap in the significantly changed genes that are upregulated between Krt16-null paw skin lesions and KRT6 human cases. (G) Overlap in the significantly changed genes that are downregulated between Krt16-null paw skin lesions and KRT6 human cases. Boxes for (D)-(G) list common genes between mouse and human data sets (listed in alphabetical order).

The top 10 most downregulated genes consist of an eclectic group with no clear theme in gene function (Fig. 1B). Followup RT-qPCR assays performed on RNA obtained from Krt16null footpad lesions for a select subset of these genes, namely myocilin (MYOC), tenascin XB (TNXB), Krt9, heat shock protein B7 (HSPB7), myozenin 2 (MYOZ2) and indolethylamine $\mathrm{N}$ methyltransferase (INMT) confirmed their downregulated status (Fig. 1C). As detailed below, the finding of markedly reduced Krt9 expression was selected for a deeper investigation because this gene is highly and specifically expressed in terminally differentiating keratinocytes of volar epidermis, and mutations in human KRT9 can cause PPK.

\section{Comparison of global gene expression in Krt16-null footpad lesions to PC patients}

The availability of a genome-wide survey of gene expression from phenotypic Krt16-null mouse footpad skin provides an opportunity to further test for the strengths and limitations of this model through a formal comparison with previously 
published microarray data sets of PPK lesions from individuals with PC (21). The human survey entailed the pairwise comparisons of PPK lesions with clinically uninvolved skin, within the same individual, for seven independent cases of PC harboring mutations in either KRT16 (three cases), KRT6 (three cases) or KRT17 (one case) (21).

To study the relationship of transcriptional changes between Krt16-null mouse footpad skin lesions and each of these KRT6 and KRT16 mutation cases, we merged the human PPK data with the mouse Krt16 data set based on human-mouse orthologs. Genes present in both data sets were preserved for further comparisons. As shown in Supplementary Material, Figure 1, there is a statistically significant and positive correlation between the transcriptional changes of Krt16-null mouse lesions and human PPK lesions, with the lowest correlation involving a K6A-N171K case $(r=0.062 ; \mathrm{P}=6.72 \mathrm{e}-16)$ and the highest correlation involving the K6B-E472K case $(r=0.19 ; P=9.81 \mathrm{e}-137)$. Besides, transcriptional changes in PPK lesions show a significant degree of correlation among PC individuals, with the highest concordance involving the two independent cases of a K16-R127C mutation $(r=0.48 ; P=0.000)$ and the lowest concordance involving the K6A-N171K case and K16-R127G cases $(r=0.13$; $P=2.54 \mathrm{e}-64)$. Two independent cases harboring a K6A-N171K mutation had a lower correlation coefficient to each other $(r=0.18$; $P=2.80 \mathrm{e}-$ 132) than to the K6B-E472K case $(r=0.29 ; \mathrm{P}=0.000$ or $r=0.19$; $P=2.67 \mathrm{e}-135)$. These findings (Supplementary Material, Fig. 1) highlight a pronounced degree of heterogeneity among cases of PC-based PPK, not only between individuals bearing different keratin mutations but also among those bearing the same mutant keratin allele (21). While the correlation coefficient $(r)$ values between the Krt16-null data set and each individual PC case may come across as low, the occurrence of a positive correlation is nonetheless significant given the broad range of correlation coefficients observed between human PC cases. Further, there are significant differences in the design of these surveys [human samples entailed the pairwise comparison between clinically involved and clinically uninvolved skin within the same individual subjects; the mouse study entailed a global comparison between Krt16-null mice $(\mathrm{N}=4)$ and WT mouse samples $(\mathrm{N}=4)$ ] as well as in the data filtering and analysis. Besides, the human cases involve markedly induced expression of KRT6 isoforms and KRT16 including the disease-causing allele (21), whereas the mouse model entails a Krt16-null mutation.

We then sought to look for genes with similar expression changes between Krt16-null paw skin lesions and PPK lesions from cases of human PC. To this end we maintained our focus on genes showing $a \geq 3$ SD change in the mouse data set (this study) and $\geq 3.5$-fold change and $P$-value of 0.1 in the human data sets (21). This strategy yielded a total of 391 upregulated and 564 downregulated genes in the mouse data with known orthologs in the human genome, 78 upregulated and 129 downregulated genes in the KRT16 human data set and 121 upregulated and 41 downregulated genes in the KRT6 human data set. Of the 391 upregulated genes from the mouse data set, 22 are shared with KRT16 human cases $(P=5.0 \mathrm{e}-19 ;$ Fig. 1D) and 32 are shared with KRT6 human cases $(P=1.4 \mathrm{e}-25$; Fig. 1F). Of the 564 downregulated genes in the mouse data set, 30 are shared with KRT16 human cases $(P=1.1 \mathrm{e}-16$; Fig. $1 \mathrm{E})$ and 9 are shared with KRT6 human cases $(P=1.2 \mathrm{e}-6$; Fig. $1 G)$.

Among the commonly upregulated genes between Krt16-null paw skin lesions and KRT6 or KRT16 cases of PC, we observe an enrichment for genes involved in barrier homeostasis (e.g. CSTA, KRT6A, S100A8, S100A9 and SERPINB2 among others) as expected based upon our previous work (11). Conversely, some of the genes that stand out among the commonly downregulated genes between Krt16-null paw skin lesions and human PPK lesions include desmin (Des), a type III intermediate filament gene important for muscle function, MYOC, a cytoskeletal protein important for maintaining intraocular pressure in the eye, and keratin 2 (KRT2), a type II intermediate filament gene restricted to differentiating keratinocytes. Of note, mice null for both Krt2 and Krt10, which both code for differentiation-specific keratins, develop a keratoderma-like phenotype on footpad skin (22). Interestingly, only the KRT6 human cases showed decreased KRT9 expression similar to the Krt16-null paw skin lesions. Full lists of these commonly changed genes between mouse and human data sets are provided in Supplementary Material, Tables 5-8. Altogether, these comparisons provide a strong case that lesional Krt16null footpad skin mimics PC-associated PPK lesions at a global gene expression level, reinforcing and extending the notion that the Krt16-null mouse is a legitimate model for the study of pathogenesis of PPK lesions associated with PC.

\section{Genetic ablation of IL-1R minimally impacts presentation of Krt16-null footpad lesions}

IL-1 signaling, a key innate immune signaling pathway, is heightened in several inflammatory cutaneous disorders, including psoriasis and atopic dermatitis (23) and can be activated by secretion of DAMPs (24). Moreover, IL-1 signaling regulates communication between epidermal keratinocytes and dermal fibroblasts during wound healing (25), and a lack of cross-talk between such cell types could account in part for the diminished (yet still significant) alarmin response to chemical challenge in Krt16-null mouse keratinocytes in primary culture relative to intact skin tissue (11). The IPA of our microarray data set predicted IL- $1 \alpha$ and IL-1 $\beta$ as top upstream regulators that could account for global gene expression changes in Krt16-null paw skin lesions (Supplementary Material, Table 3). Further, several IL-1 family signaling molecules are elevated in Krt16-null footpad lesions (Supplementary Material, Table 4) (11). Such evidence that IL-1 signaling could be a major player in PPK pathogenesis prompted us to evaluate the potential that K16 interfaces with IL-1R-dependent signaling in volar skin.

We crossed Krt16-null with IL-1R-null mice (both in the $\mathrm{C} 57 \mathrm{Bl} / 6$ inbred strain background), in which IL-1-dependent signaling is abrogated (26). All IL-1R;Krt16 double-null male mice spontaneously developed PPK-like footpad lesions between 4 and 6 weeks of age (Supplementary Material, Fig. 2A), with minimal difference in the average date of lesion onset between genotypes (Krt16 null: $33.1 \pm 1.8$ days; IL-1R;Krt16 double null: $35.9 \pm 2.0$ days; $P=0.49$ ). Macroscopically, the PPK-like lesions still formed preferentially on pressure points of footpad skin and appeared as hyperpigmented calluses (Supplementary Material, Fig. 2B). Quantitation of these lesions via the PPK index, an objective measure of their severity (blind assessment; see Materials and Methods), showed that there was no difference between genotypes (Krt16 null: 20.3 \pm 8.3 ; IL-1R;Krt16 double null: $26.5 \pm 6.9 ; \quad P=0.60$ ) (Supplementary Material, Fig. 2C). Histologically, IL-1R;Krt16 double-null mice showed a similar amount of epidermal thickening relative to the Krt16null reference (Supplementary Material, Fig. 2D; quantitation reported in Supplementary Material, Fig. 2E). At a molecular level, upregulation of DAMP-encoding mRNA transcripts 
including Sprr2d, Krt6a, DefB4, S100A9 and SerpinB3 was also similar in IL-1R;Krt16 double-null compared to Krt16-null paw skin (Supplementary Material, Fig. 2F). These findings thus strongly suggest that IL-1R-dependent signaling does not play a significant role in the pathophysiology of PC-like PPK lesions in Krt16-null footpad skin.

\section{Decreased Krt9 expression precedes onset of lesions in Krt16-null paw skin}

Among the most downregulated genes in Krt16-null paw skin is Krt9, which stands out because it codes for a type I keratin and represents the most abundant mRNA transcript expressed in human volar skin (27). Further, KRT9 is mutated in the setting of human EPPK (28) and a null mutation at the Krt9 locus gives rise to an EPPK-like phenotype in mouse, consisting of epidermal thickening, hyperkeratosis, hyperproliferation, impaired terminal differentiation and suprabasal cell lysis (13). Other researchers reported that KRT9 transcript (21) and K9 protein (21, 29) are frequently downregulated in PPK lesions of individuals with PC $(21,29)$. These elements together pointed to a potential role for the loss of Krt9 expression in the pathophysiology of the PPK-like lesions arising in Krt16-null footpad skin and to a possible defect in terminal differentiation in this setting.

We sought to confirm and extend the microarray-based finding of reduced Krt9 expression at 2 months of age in Krt16-null footpad skin. We analyzed Krt9/K9 expression using molecular assays and tissue immunostaining in 2 month, 1 month and 2 week old mice, respectively, corresponding to 'late', 'early' and 'pre' stages of PPK (17) and also in E18.5 embryos, immediately before birth. By RT-qPCR assays, the Krt9 mRNA was reduced by $\sim 50 \%$ at 2 weeks and by $>90 \%$ at 4 weeks and 8 weeks after birth in Krt16-null relative to WT footpad skin (Fig. 2A). Interestingly, in late stage (E18.5) embryos where $\mathrm{Krt9}$ is expressed at low levels in WT paw skin (average E18.5 Cq $=29.6$ versus 2 weeks $\mathrm{Cq}=26.6$ ), the Krt9 mRNA is already reduced by $60 \%$ in Krt16-null relative to WT (average WT Cq $=29.6$ versus Krt16-null Cq $=30.5$ ) (Fig. 2A). By western immunoblotting, the $\mathrm{K} 9$ protein was reduced by $\sim 50 \%$ at 2 weeks and 4 weeks and by $\sim 90 \%$ at 8 weeks after birth in Krt16-null relative to WT footpad skin (Fig. 2C; see Fig. 2B for quantitation). Indirect immunofluorescence confirmed the dramatic reduction in $\mathrm{K} 9$ expression and showed that the loss occurs across the entire suprabasal epidermis in 2 week old Krt16-null footpad skin relative to WT controls (Fig. 2D). Thus, the loss of Krt9/K9 occurs very early in Krt16-null footpad skin, well before the onset of the lesion process, raising the dual prospect that it could reflect impaired differentiation and play a role in PPK pathophysiology.

\section{Terminal differentiation is altered ahead of onset of PPK-like lesions in Krt16-null footpad skin}

Histologically, paw skin tissue of 2 week old Krt16-null mice show minor alterations relative to WT littermates. At high magnification, granular layer keratinocytes are misshapen while basal keratinocytes appear crowded and their nuclei are less columnar in shape, as conveyed by a statistically significant decrease in the aspect ratio (WT: $2.2 \pm 0.1, \mathrm{Krt16}$ null: $1.8 \pm 0.1 ; \mathrm{P}=0.005$ ) (Fig. 3A and B). Given the Krt9 findings (Fig. 2), this prompted us to survey additional markers of epidermal differentiation. The transcript levels for Krt1, Krt10 and involucrin (Ivl), which mark an early stage of differentiation, and those for Krt2, filaggrin (Flg), and loricrin (Lor), which reflect a later stage of differentiation, were all significantly elevated in 2 week old Krt16-null front paws (Fig. 3C). Such molecular attributes suggest that an effort to stimulate terminal differentiation gets under way in Krt16-null footpad skin, significantly ahead of the development of PPK-like lesions. Of note, several of the differentiation markers elevated at 2 weeks of age are not significantly changed in Krt16-null paw skin relative to controls at E18.5 (Fig. 3D).

\section{Loss of Krt9/K9 expression is likely not the result of the Krt16 targeting strategy}

Type I keratin genes are clustered and organized in a conserved fashion in mammalian genomes (30). On mouse chromosome 11 (31) the Krt9 locus is located $52.8 \mathrm{~kb}$ pairs away from the Krt16 locus, with the Krt14 gene located in between (Fig. 4A). Such proximity raises the prospect that introduction of a null mutation at the Krt16 locus, which was achieved by replacement of the open reading frame with a LacZ cassette as part of the knock-out mouse project (KOMP) (10), would impact the regulation of Krt9 and other proximal genes in the locus. To evaluate this possibility, we measured the steady state mRNA levels for Krt15, Krt14, Krt17 and Krt42 in Krt16-null and WT mouse footpad skin at 2 weeks, 4 weeks and 8 weeks of age. The Krt15 mRNA is elevated at 2 and 4 weeks of age in Krt16-null footpad skin but is comparable to WT at 8 weeks of age (Fig. 4B). The Krt14 mRNA is transiently decreased at 4 weeks of age in Krt16-null footpad skin, but remains similar to WT at 2 and 8 weeks of age (Fig. 4B). Interestingly, the Krt17 mRNA is decreased at 2 and 4 weeks in Krt16-null footpad skin, but dramatically increases at 8 weeks of age in Krt16-null paw skin (Fig. 4B), consistent with its well-known induction in lesional and inflamed skin (32). Lastly, the Krt42 mRNA is increased at 2 and 8 weeks of age but is decreased in 4 weeks in Krt16-null relative to WT footpad skin (Fig. 4B). Therefore, Krt9 is the only gene in this region of the type I keratin gene cluster whose transcript is significantly and consistently decreased at all three time points, implying that the cause of the loss of Krt9 expression is likely not an artifact of locally disrupting the Krt16 locus. Besides, and as reported below, the small molecule-based prevention of the footpad skin phenotype in Krt16-null mice entails a robust induction of the Krt9/K9 expression.

As part of this analysis, we also examined the transcript levels for Krt6a, which is considered the natural type II keratin partner gene for Krt16 (33), and Krt5, the main partner gene for Krt14. The Krt6a mRNA is significantly increased in Krt16null paw skin at 2, 4 and especially in 8 week old Krt16-null mice relative to WT footpad skin (Fig. 4C), consistent with its highly stress-inducible character (34). The extent of its induction increased along time and reached very high levels $(>12$-fold increase over WT) in established PPK-like lesions of Krt16-null mice. Of interest, KRT6a expression is markedly elevated in PPK lesions of individuals with PC, though it is accompanied by a parallel increase in KRT16 expression in such cases $(11,21)$. The mRNA levels of Krt5 prevailing at 2, 4 and 8 weeks are quite stable in Krt16-null and WT footpad skin (Fig. 4C), further highlighting the differential regulation of highly similar and proximally located keratin genes in this setting.

\section{SF-mediated prevention of the PPK-like lesions entails Krt9 induction in Krt16-null skin.}

We previously reported that timely topical treatment of male Krt16-null mice with SF, a small molecule inducer of Nrf2-Keap1 

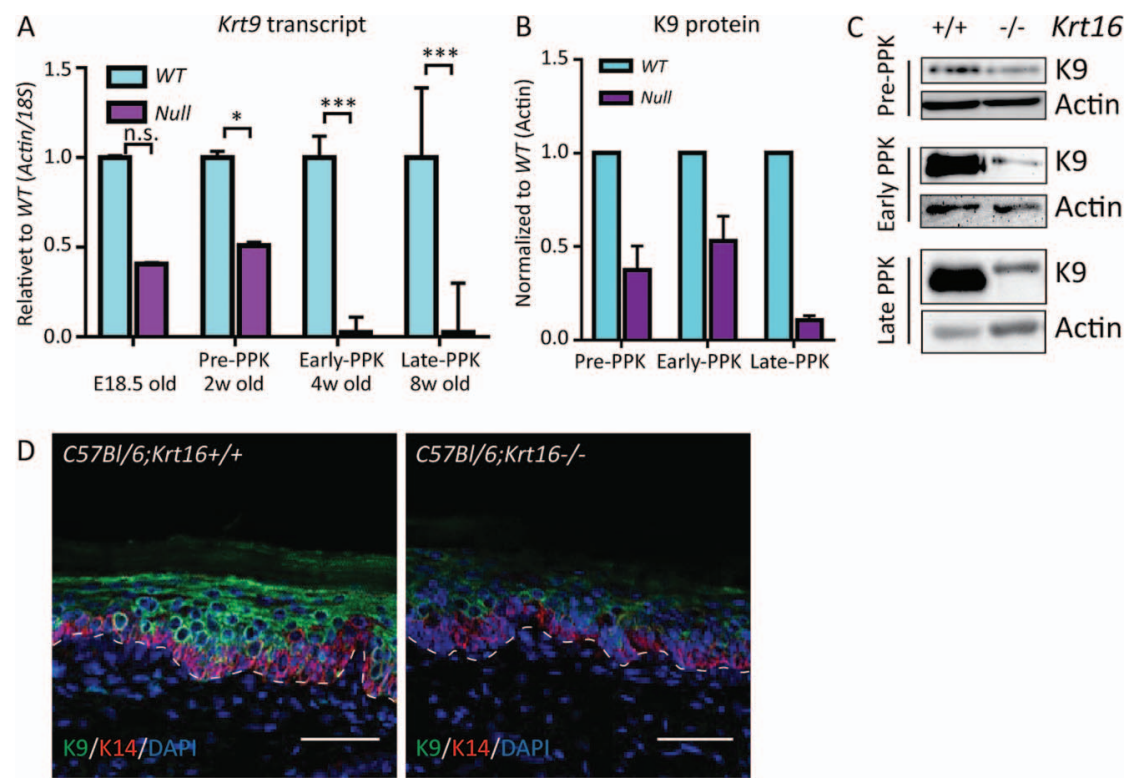

Figure 2. Decreased Krt9 expression precedes footpad lesion onset. (A) Transcript levels of Krt9 at various points in Krt16-null footpad lesion progression. $\mathrm{N}=4-$ $8 \mathrm{mice} /$ genotype/time point. (B) Quantitation of total $\mathrm{K} 9$ protein at various points in Krt16-null footpad lesion progression. $\mathrm{N}=2-6$ mice/genotype/time point. (C) Representative western blots for total K9 protein in Krt16-null relative to WT controls. Actin was used as a loading control. (D) Confirmation of decreased K9 protein 2 weeks prior to footpad lesion onset in Krt16-null paw skin by immunofluorescence staining. Scale bar, $50 \mu \mathrm{m}$. All data presented as mean fold change relative to WT + SEM. ${ }^{*} P<0.05,{ }^{* *} P<0.01,{ }^{* * *} P<0.001$. n.s. denotes not significant.

A

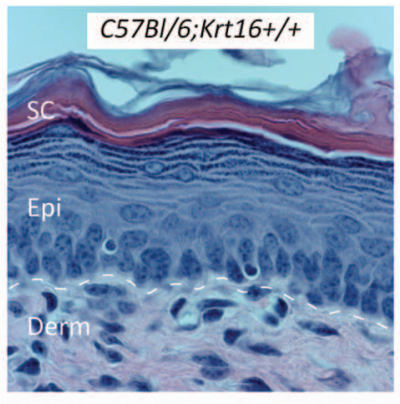

2 week-old paw skin

C

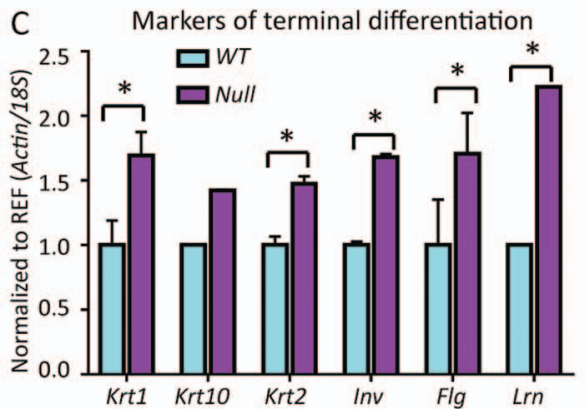

C57BI/6;Krt16-/-

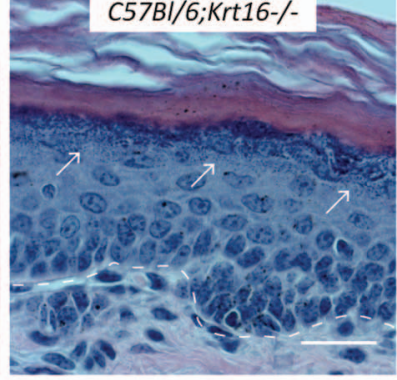

B

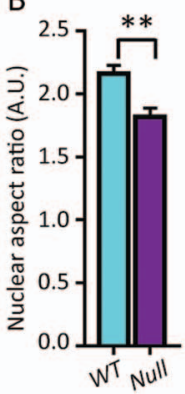

E18.5 paw skin

D Markers of terminal differentiation

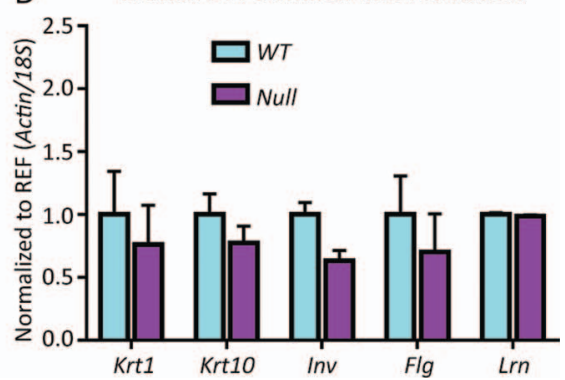

Figure 3. Impaired epidermal differentiation of volar keratinocytes in 2 week old Krt16-null paw skin. (A) H\&E staining of paw skin from Krt16-null and WT littermate control at 2 weeks of age. Scale bar, $50 \mu \mathrm{M}$. SC, stratum corneum; Epi, epidermis; Derm, dermis. Dermal/epidermal junction denoted by dotted line. Arrows highlight abnormalities in granular layer keratinocytes. (B) Quantitation of nuclear aspect ratio of basal keratinocytes in volar epidermis. (C) Transcript levels of multiple markers of terminal differentiation at 2 weeks of age in Krt16-null relative to WT paw skin. (D) Transcript levels of multiple markers of terminal differentiation at E18.5 in Krt16-null and WT paws. $\mathrm{N}=4-8$ mice/genotype. All data presented as mean fold change relative to WT $+\mathrm{SEM} .{ }^{*} \mathrm{P}<0.05,{ }^{* *} \mathrm{P}<0.01,{ }^{* * *} \mathrm{P}<0.001$.

signaling (35), prevents the development of PPK-like lesions in footpad skin (17). Whereas this SF treatment alone is not effective in female Krt16-null mice, addition of the ER- $\beta$ agonist DPN enables proper activation of Nrf2 and prevents the development of footpad lesions (18). We next tested whether the prevention of PPK-like lesions in SF-treated Krt16-null mice includes the restoration of $\mathrm{Krt9} / \mathrm{K} 9$ expression in footpad skin. Male mice treated topically with SF ahead of footpad lesion onset showed successful activation of Nrf2 along with restoration of Krt9/K9 expression (Fig. 5A-E). This treatment regimen did not 
A Ensembl Mus musculus version 90.38 Chromosome 11: 100,116,436-100,302,347
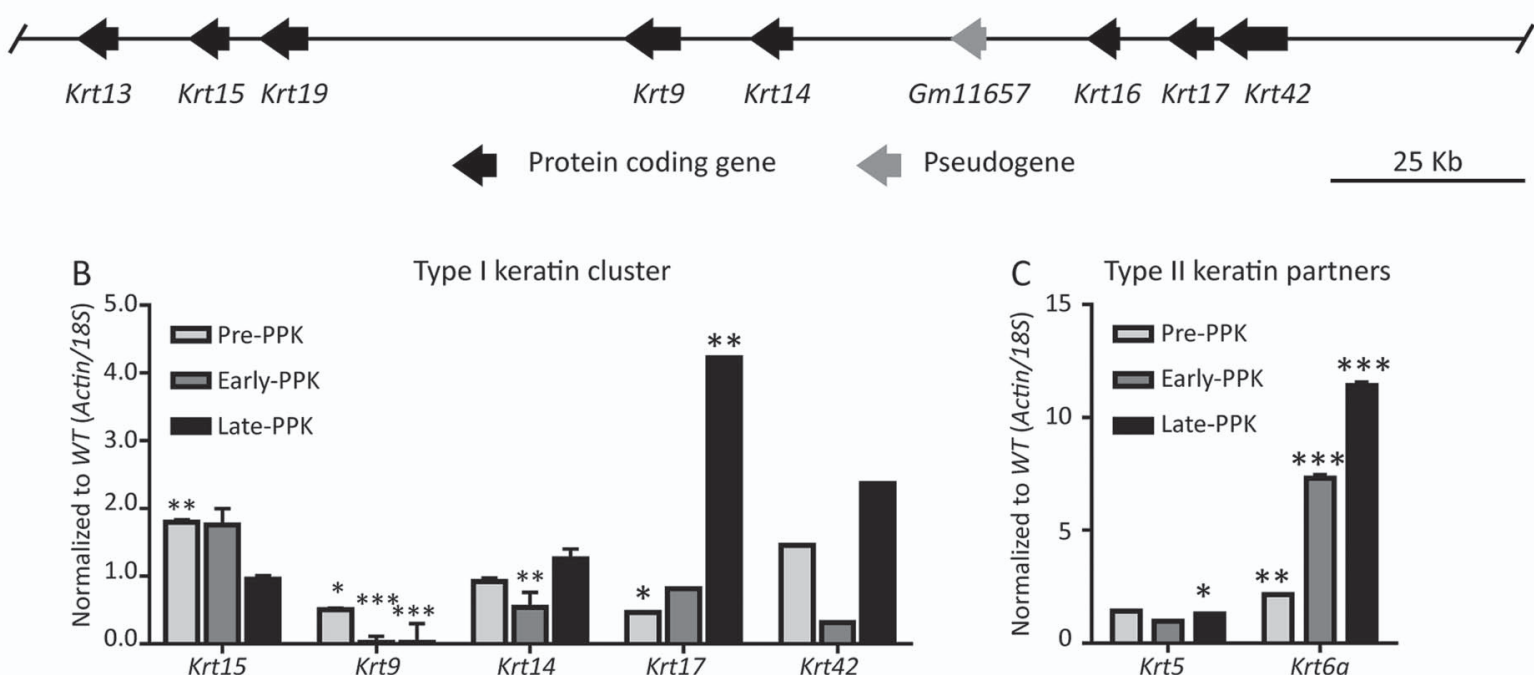

Figure 4. Modest fluctuations in Krt16 proximal genes in Krt16-null mice throughout footpad lesion progression. (A) Schematic of the mouse type I keratin cluster. (B) Transcript levels of several skin keratins throughout Krt16-null footpad lesion progression. (C) Transcript levels of type II keratin partners K5 and K6 throughout Krt16-null footpad lesion progression. $\mathrm{N}=4-8$ mice/genotype/time point. All data presented as mean fold change relative to WT $+\mathrm{SEM}$. ${ }^{*} \mathrm{P}<0.05,{ }^{* *} \mathrm{P}<0.01$, ${ }^{* * *} \mathrm{P}<0.001$.

succeed in activating Nrf2 signaling in female Krt16-null mice, or in male mice for which the treatments started after lesion onset, and there was no rescue of Krt9/K9 levels in any such cases (Fig. 5A-E). While these observations do not formally rule out that the restoration of $\mathrm{Krt9} / \mathrm{K} 9$ expression is a bystander effect of SF treatment, they do suggest that the ability of SF to prevent PPK-like footpad lesions in Krt16-null mice involves the restoration of $\mathrm{Krt9} / \mathrm{K} 9$ expression, implying that loss of the latter plays a role in the pathophysiology of the lesions arising in Krt16null footpad skin.

\section{Krt9 expression is not directly regulated by Keap1-Nrf2 signaling}

Oxidative stress secondary to hypoactive Keap1-Nrf2 signaling occurs at the time of lesion onset and represents a key driver of the Krt16-null PPK-like phenotype (17). Further, Nrf2 signaling is known to stimulate terminal differentiation in epidermis and esophagus $(36,37)$ and promote barrier function in skin $(38,39)$, raising the prospect that this pathway could be directly inducing Krt9 expression. Nrf2 directly regulates expression of keratins 6 and 16 through functional antioxidant response elements (AREs) (40). Analysis of genomic DNA flanking the KRT9 (human) and Krt9 (mouse) loci revealed the presence of several putative AREs proximal to the coding sequence (Supplementary Material, Fig. 3), further supporting the notion that Nrf2 signaling may play a role in regulating Krt9 expression in volar epidermis.

We find that both the Nrf2 transcript and protein are statistically within normal levels at 2 weeks of age in Krt16-null paw skin (Fig. 6A-C), while that of its target genes Nqo-1, HO-1 and Sprr2d are modestly upregulated (Fig. 6D). Further, there is no statistically significant change in transcript levels of several genes involved in the synthesis and metabolism of the major antioxidant glutathione including glutathione synthetase (GS), glutamine cysteine ligase catalytic subunit (GCLC), glutathione reductase (GSR) and glucose-6-phosphate dehydrogenase (G6PD) (Fig. 6E). This is in contrast to what was observed at 4 weeks of age just prior to lesion onset, when there is a significant decrease in transcript and protein levels for both GS and GCLC (17). To build on these findings, we measured the levels of the major antioxidant glutathione, which occurs as either its reduced (GSH) or oxidized form (GSSG). There was no significant difference in total glutathione, GSH, or GSSG levels between Krt16 WT, heterozygous or null mouse paw skin at 2 weeks of age (Fig. $6 \mathrm{~F}-\mathrm{H})$, again unlike the situation that prevails at 1 month of age (17). We then evaluated Nrf2-null paw skin at 4 weeks of age and found that there is a 2-fold increase in the Krt9 transcript levels, which is statistically significant and likely reflects altered regulation of terminal differentiation, along with a very modest increase in $\mathrm{K} 9$ protein levels (Fig. 6I-K). These findings indicate that Nrf2 is not essential for Krt9 transcription in the epidermis of volar skin, in spite of the presence of predicted AREs in the locus (41). Further, they suggest that Keap1-Nrf2 signaling does not play a direct role in reduced levels of Krt9/K9 occurring in Krt16-null skin and otherwise suggest that terminal differentiation is altered ahead of a state of oxidative stress in this model.

\section{Assessing the status of defined pathways regulating terminal epithelial differentiation in skin}

We next investigated the status of pathways known to impact terminal differentiation in an effort to determine the basis for loss of $\mathrm{Krt9} / \mathrm{K} 9$ expression under circumstances where terminal differentiation seems to be stimulated. The literature suggests that expression of Krt9 is regulated in part by epithelial-mesenchymal interactions. Co-culture of nonvolar keratinocytes with volar skin-derived fibroblasts can induce Krt9/K9 expression (42). The dermal transcription factor Homeobox A13 (HOXa13) regulates the development of distal appendages, and mice deficient for HOXa13 do not express WNT5a or Krt9 in palmoplantar keratinocytes (43). Of note, defective Krt9 expression in HOXa13 deficient mice can be rescued by restoring WNT5a expression (43). We found that transcript levels for HOXa13 were similar in WT and Krt16-null footpad skin. By contrast, the transcripts for two established 


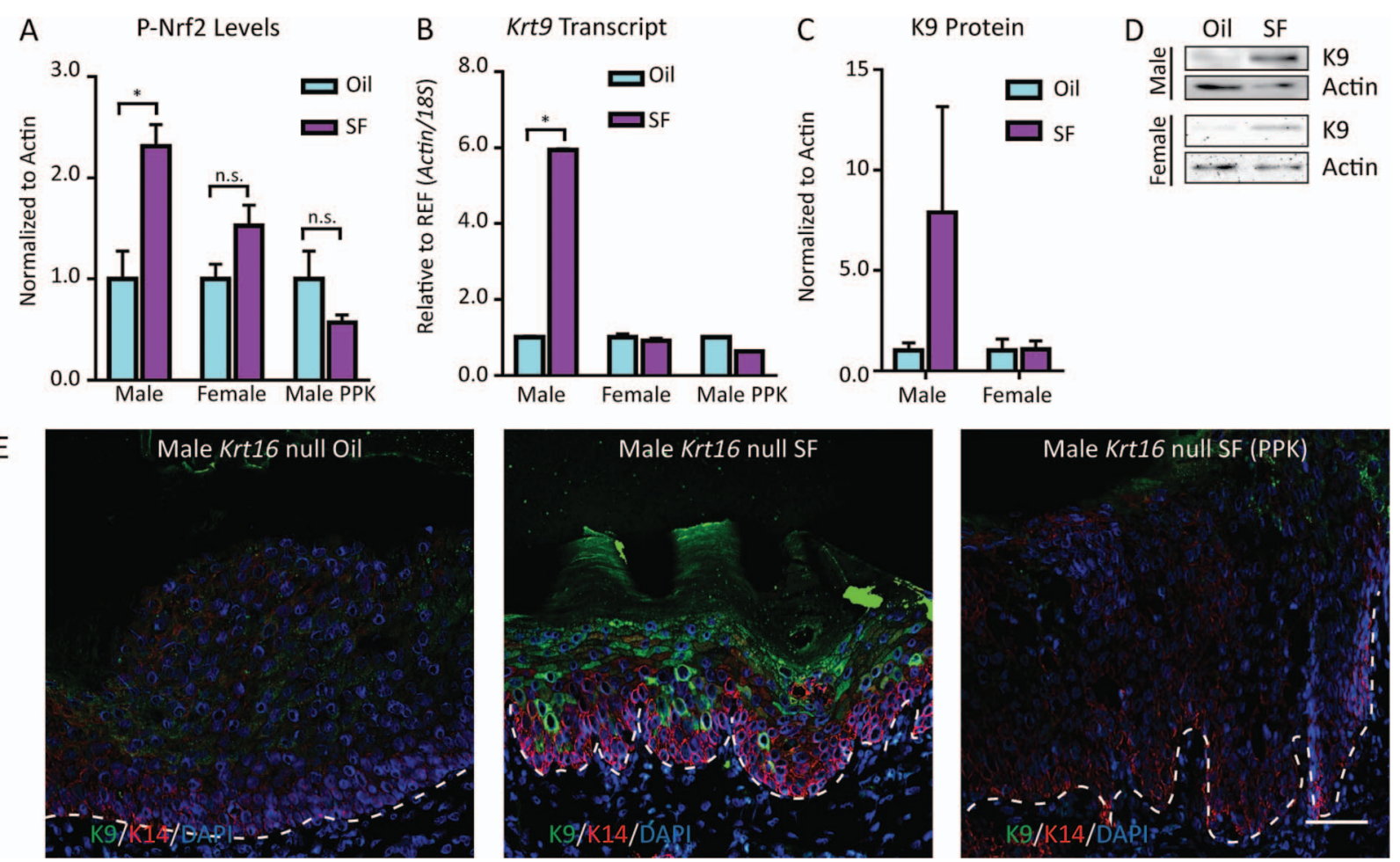

Figure 5. SF treatment induces K9 expression in male Krt16-null paw skin. (A) Quantitation of P-Nrf2 immunostaining in male and female Krt16-null paw skin treated topically with either SF or Oil. $\mathrm{N}=2-4$ mice/treatment/sex. Data presented as mean fold change + SEM. Male PPK refers to male Krt16-null mice treated topically with SF after the appearance of footpad lesions. ${ }^{*} \mathrm{P}<0.05$. n.s. denotes not significant. (B) Transcript levels of Krt9 in Krt16-null mice treated with SF relative to vehicle control (Oil). $\mathrm{N}=4-6$ mice/treatment/sex. Data presented as mean fold change + SEM. ${ }^{*} P<0.05$. (C) Quantitation of total K9 protein from western blot analysis of protein lysates of front paws from male and female Krt16-null mice with either SF or Oil treatment. $\mathrm{N}=4$ mice/treatment/sex. Data presented as mean fold change + SEM. (D) Representative western blots of K9 protein in front paw lysates from male and female Krt16-null mice treated with either SF or Oil. Actin was used as a loading control. (E) Fresh frozen paw sections from SF or Oil treated male Krt16-null mice both prior as well as after onset of footpad lesions immunostained for K9. $\mathrm{N}=2$ mice/treatment. Scale bar, $50 \mu \mathrm{M}$.

HOXa13 target genes, ephrin tyrosine kinase A7 (EphA7) and bone morphogenetic protein 2 (BMP2), were significantly upregulated in 2 week old Krt16-null relative to WT footpad skin (Fig. 7A). From this, we infer that HOXa13 activity may be upregulated and thus is not likely to account for diminished Krt9 transcript levels in Krt16-null paw skin.

WNT signaling also regulates Krt9 expression via epithelialmesenchymal interactions. While there are reports that inactivation of WNT signaling by the inhibitor Dickkopf 1 , which is enriched in palmoplantar keratinocytes, can stimulate Krt9 expression $(44,45)$, more recent data conveys that KRT9 expression can be induced in keratinocyte-only cultures by activation of WNT signaling (27). We find that the transcript levels for canonical WNT ligands Wnt10a and Wnt10b are significantly increased in Krt16-null paw skin, whereas the noncanonical WNT ligand Wnt5a mRNA is minimally impacted (Fig. 7B). Elevated transcript levels for Axin2 and the receptor Frizzled 8 (FZD8), which are established WNT target genes, provide further evidence that canonical WNT signaling is also possibly elevated in Krt16-null paw skin (Fig. 7B).

We next assessed the status of pathways anchored by Notch and Distal-Less Homeobox 3 (Dlx3). Notch signaling promotes keratinocyte differentiation under normal circumstances and absence of Notch1 causes aberrant keratinocyte differentiation (46). Transcript levels for Notch1, Jagged1 and Hes1 are modestly increased in Krt16-null paws at 2 weeks of age, with only Notch1 and Jagged1 reaching statistical significance (Fig. 7C). Keratinocytes deficient for the transcription factor Dlx3 exhibit upregulated DAMPs expression upon challenge (47), similar to Krt16-null skin (11). Yet, the transcript levels for Dlx3 and two of its target genes, Desmoplakin (Dsp), and Envoplakin (Evpl), were significantly upregulated in Krt16-null paws at 2 weeks of age (Fig. 7D), again making it unlikely that the loss of Krt9 is due to impaired Dlx3 signaling.

Mechanical stress is yet another driver of terminal epidermal differentiation (48-50) and is poised to play a key role in palmar/plantar epidermis, a tissue that experiences mechanical stress. Hippo/YAP1 (Yes Associated Protein 1) is a mechanosensitive pathway and has been reported to negatively regulate epidermal differentiation by promoting stem cell proliferation and antagonizing Notch signaling (51,52). At 2 weeks of age, we observe modest upregulation of the YAP1 target genes cysteine rich angiogenic inducer 61 (Cyr61), connective tissue growth factor (Ctgf), zinc finger E-box binding homeobox 1 (Zeb1), and Snail in Krt16-null paw skin relative to control, which do not reach statistical significance (Fig. 7E). Finally, aryl-hydrocarbon receptor (AhR) signaling is another stress response pathway involved in skin barrier homeostasis $(53,54)$. Similar to Nrf2, AhR is sequestered in the cytoplasm and, upon activation, translocates to the nucleus to promote transcription of xenobiotic metabolism genes. Here we found that the AhR target genes cytochrome P450 1A1 (Cyp1a1) and Cyp1b1 were each significantly increased in Krt16-null paw tissue at 2 weeks of age, whereas aldehyde dehydrogenase 3A1 (ALDH3A1) and UDP glucuronosyltransferase 1A2 (UGT1A2) were minimally impacted (Fig. $7 F$ ). In summary, the nature of the alterations 
2 week-old paw skin (Krt16 mice)
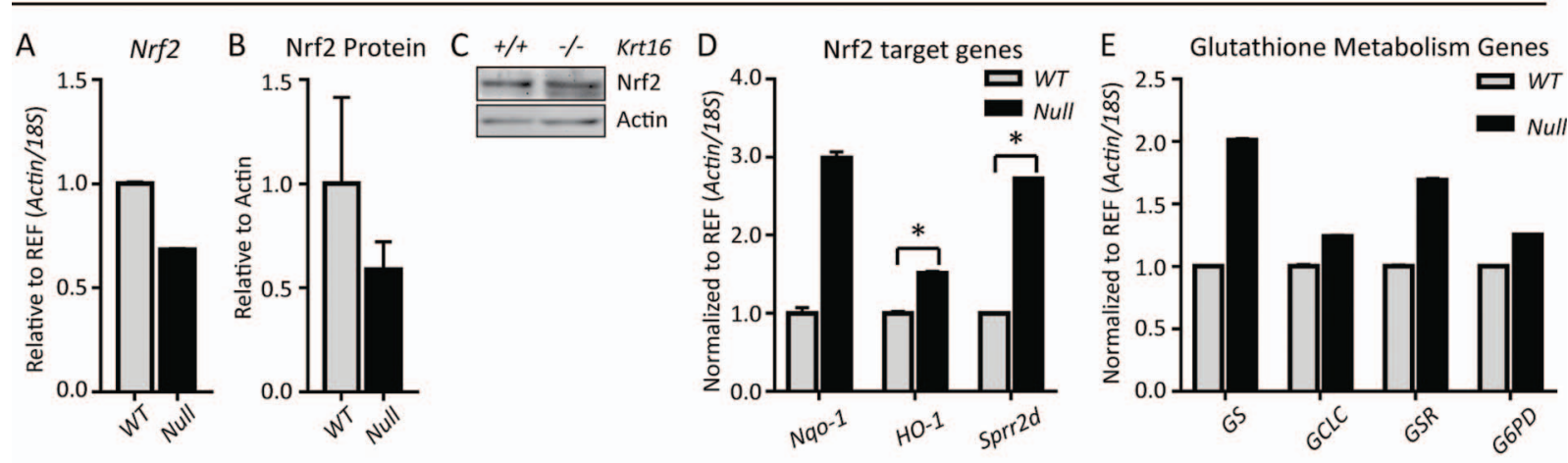

2 week-old paw skin (Krt16 mice)
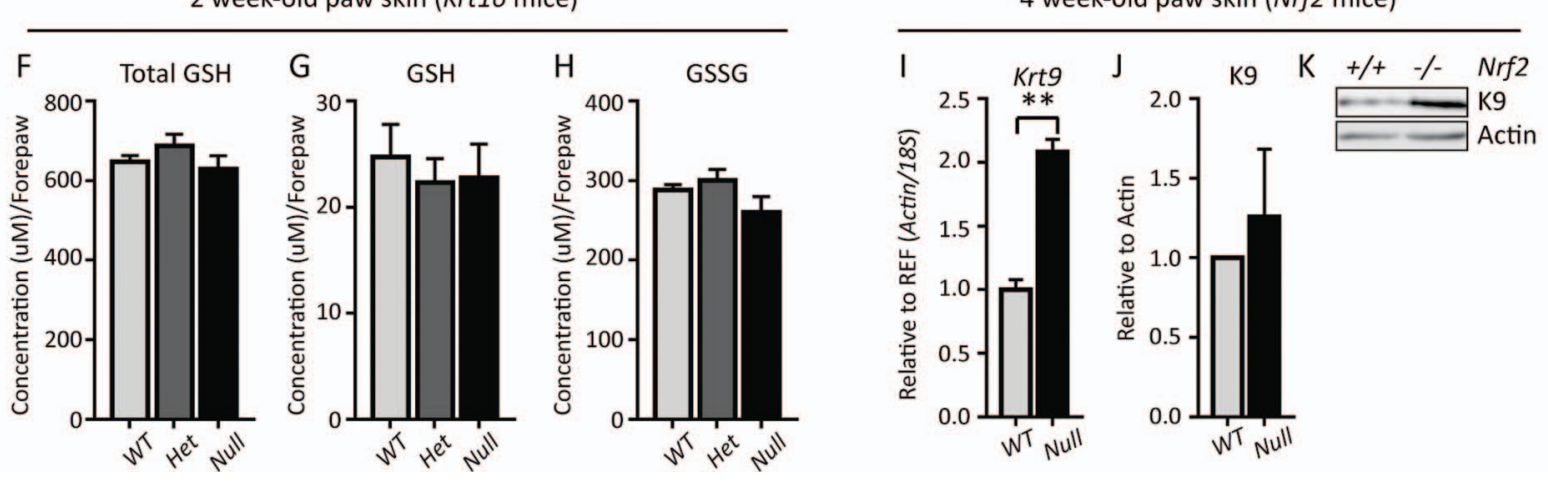

Figure 6. Role of Keap1-Nrf2 signaling in Krt9 expression. (A) Nrf2 transcript levels at 2 weeks of age in Krt16-null front paws. N = 6-8 mice/genotype. (B) Quantitation of total Nrf2 protein in Krt16-null paw skin as assessed by western blotting at 2 weeks of age. $\mathrm{N}=6$ mice/genotype. (C) Representative western blot for total Nrf2 protein from WT and Krt16-null paw skin. Actin was used as a loading control. (D) Transcript levels of Nrf2 target genes NADPH quinone dehydrogenase 1 (Nq01), Heme Oxygenase-1 (HO-1) and Sprr2d at 2 weeks of age in Krt16-null front paws. N = 6-8 mice/genotype. (E) Transcript levels of glutathione metabolism genes GS, GCLC, GSR and G6PD at 2 weeks of age in Krt16-null front paws. $\mathrm{N}=6-8$ mice/genotype. ( $\mathrm{F}$ ) Concentration of total glutathione present in Krt16 front paws at 2 weeks of age. $\mathrm{N}=2-5$ mice/genotype. (G) Concentration of GSH present in Krt16 front paws at 2 weeks of age. N = 2-5 mice/genotype. (H) Concentration of GSSG present in Krt16 front paws at 2 weeks of age. $\mathrm{N}=2-5$ mice/genotype. (I) Krt9 transcript levels in Nrf2-null paw skin at 1 month. $\mathrm{N}=5-9$ mice/genotype. (J) Quantitation of total K9 protein in Nrf2-null paw skin as assessed by western blotting. $\mathrm{N}=5$ mice/genotype. (K) Representative western blot for total K9 protein from WT and Nrf2-null paw skin. Actin was used as a loading control. All data is presented as mean fold change relative to control + SEM. ${ }^{*} \mathrm{P}<0.05$. ${ }^{* *} \mathrm{P}<0.01$.

observed in several pathways known to impact terminal differentiation is not readily compatible with a role in the robust and sustained loss of Krt9/K9 expression in Krt16-null skin.

\section{Nuclear localization of K16}

Until this point, we assumed that K16 participates in the regulation of gene expression, whether negatively (e.g. DAMPs) or positively (Krt9) by regulating a canonical signaling pathway such as IL-1R- or Keap1-Nrf2-dependent signaling. However, emerging evidence indicates that keratins are able to regulate gene expression through interacting with transcription factors within the nucleus of epithelial cells $(55,56)$. This raised the intriguing possibility that $\mathrm{K} 16$ could potentially localize and function within the nucleus, accounting in part for its ability to impact gene expression. In support of this possibility, transgenic mice overexpressing human K16 exhibited nuclear accumulation of $\mathrm{K} 16$ in skin keratinocytes, in a non-distinct punctate form, at the ultrastructural level (57). While originally thought to be an artifact of overexpressing human K16 in a transgenic mouse model $(57,58)$ this observation raises the possibility that K16 can localize and potentially function within the nucleus.

To assess whether keratin 16 (K16) is able to localize to the nucleus, we transiently expressed GFP-tagged mouse K16
(GFP-mK16) in HeLa cells and treated them with Leptomycin B (LMB), an inhibitor of exportin 1 that has been used to facilitate the detection of nuclear-localized keratins $(55,56,59,60)$. Using laser scanning confocal microscopy, K16 puncta were visualized within the plane of the nucleus in $\sim 20 \%$ of transfected cells after treatment with LMB (data not shown). Co-treatment with the pro-inflammatory molecules 12-O-tetradecanoylphorbal-13acetate (TPA) and tumor necrosis factor (TNF) increased this frequency to $\sim 50 \%$ of transfected cells (Fig. 8A). These distinct K16 puncta do not appear to be restricted to a specific region of the nucleus, and a single nucleus can have between 2 and 15 small K16-positive puncta in such preparations. Transfecting the human, rather than mouse, $\mathrm{K} 16$ coding sequence gave rise to similar findings (66\% of transfected cells showed nuclear K16) (Fig. 8B). We next transfected A431 keratinocytes with the GFPmK16 and treated the cultures with LMB, TPA and TNF. Nuclear accumulation of $\mathrm{K} 16$ was again observed in $20 \%$ of transfected cells (Fig. 8A), indicating this behavior occurs in several species and cell lines.

$\mathrm{K} 17$, a type I keratin similar to $\mathrm{K} 16$, is robustly expressed in HeLa cells where it can occur in the nucleus (55). We next tested whether K16 nuclear accumulation was dependent upon K17 by transfecting HeLa cells carrying a Crispr-CAS9-generated null allele for KRT17 (K17 KO) (55). Unexpectedly, there was a dramatic increase in the percentage of transfected cells with 

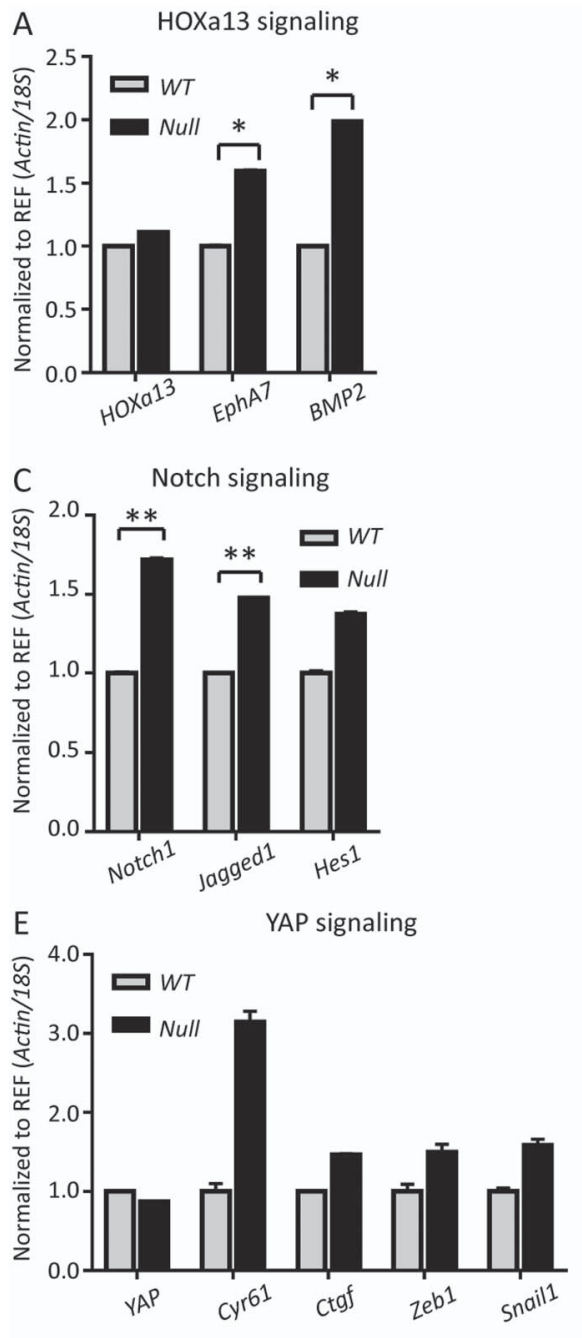
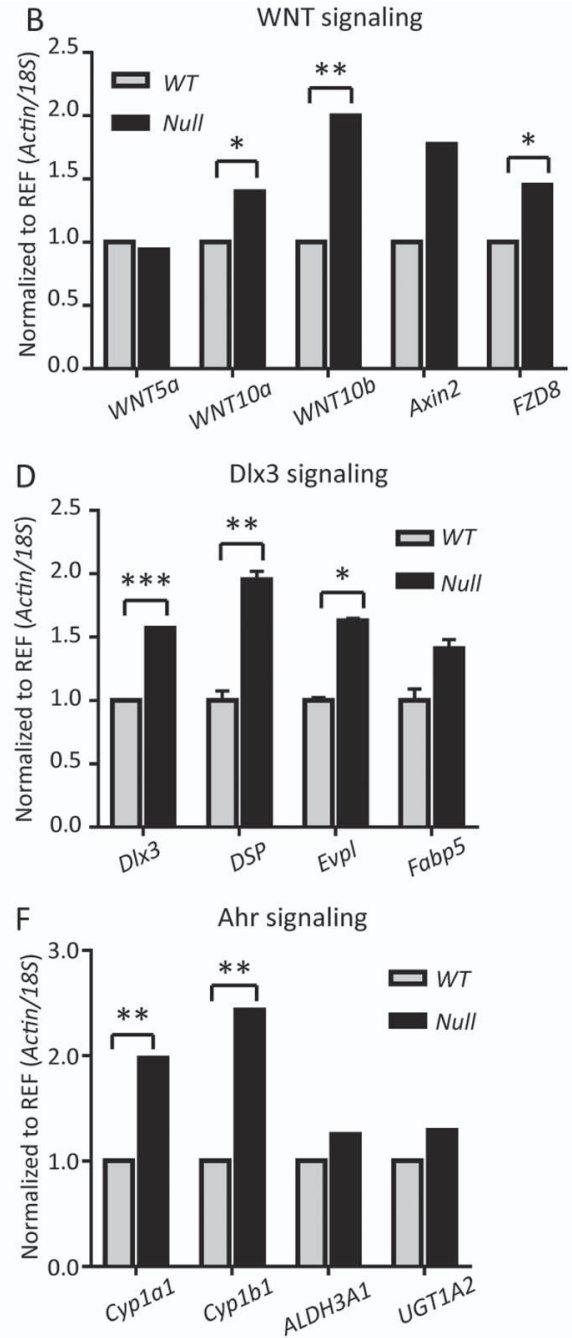

Figure 7. Assessing the status of mainstream pathways regulating terminal epithelial differentiation in skin. (A-F) Transcript levels of several effectors of terminal differentiation in Krt16-null paw skin relative to WT controls at 2 weeks of age. (A) HOXa13 signaling. (B) WNT signaling. (C) Notch signaling. (D) Dlx3 signaling. (E) Hippo/YAP1 signaling. (F) Aryl hydrocarbon signaling. $\mathrm{N}=6-8$ mice/genotype. All data presented as mean fold change relative to WT $+\mathrm{SEM}$. ${ }^{*} \mathrm{P}<0.05,{ }^{* *} \mathrm{P}<0.01$, $* * * P<0.001$.

K16 nuclear puncta in K17 KO cells (HeLa parental: 45.8\% \pm 1.7 ; HeLa KO: $94.7 \% \pm 5.3 ; P=0.013$ ) (Fig. $8 C-D$ ). This indicates that the presence of $\mathrm{K} 17$ is not necessary for and may even partially inhibit the nuclear accumulation of K16. Of further interest, when both $\mathrm{K} 16$ and $\mathrm{K} 17$ are present in the same nuclei, they form distinct puncta and occupy different regions of the nucleus (Fig. 8E).

To establish that the presence of nuclear K16 was not an artifact of transient transfection of cells in culture and investigate whether there could potentially be a role for nuclear K16 in PPK pathogenesis, we turned to Krt9-null paw skin lesions (13). K16 expression was reported to be elevated in Krt9-null paw skin lesions (13), a finding that we confirmed in our laboratory (Joseph Shen, unpublished data). Sections of paw skin lesions from 2 month old Krt9-null mice were immunostained for K16. Endogenous K16 expression was expressed throughout the entire epidermis, and a subset of cells $(<1 \%)$ distinctly showed nuclear puncta positive for K16 (Fig. 8F). We repeated this analysis for ear skin tumor samples obtained from HPV16 transgenic mice (61) and found that a subset of cells $(<1 \%)$ distinctly showed nuclear puncta positive for K16 (Fig. 8G). This suggests that endogenous $\mathrm{K} 16$ can localize to the nucleus in skin tissue in situ, in the absence of agents that alter nuclear import/export.

\section{Discussion}

Both PC as an inherited condition and PPK as a clinical manifestation are known to be highly heterogeneous. Formally assessing the degree of correlation in transcriptional changes occurring in PC cases arising from KRT6 or KRT16 mutations provided confirmation of the heterogeneity in PC-based PPK lesions between individuals. Additionally, comparing these PPK data sets with a microarray-based survey of gene expression in Krt16-null paw skin lesions provided definitive evidence that the latter undergo transcriptional changes that are similar to those occurring in PCassociated PPK lesions, solidifying its status as a relevant model for the study of pathogenesis of this condition. Evidence for this convergence is apparent in spite of fundamental differences in the way the gene expression surveys were conducted for the mouse model (comparison of WT and mutant mice) versus the human cases (comparison of clinically involved vs. non-involved 
A
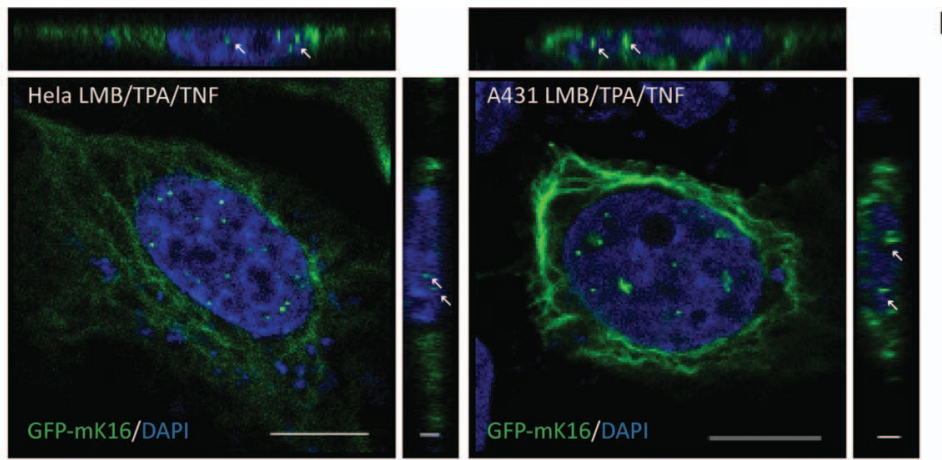

B

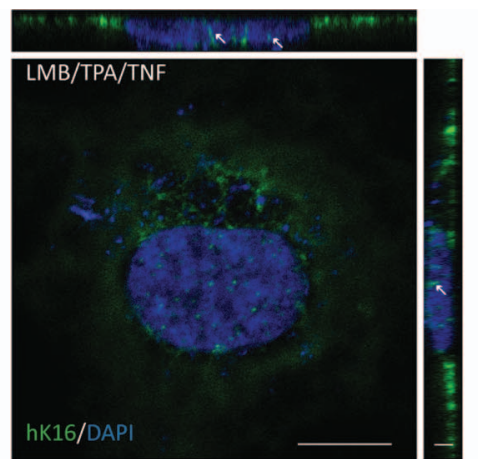

C
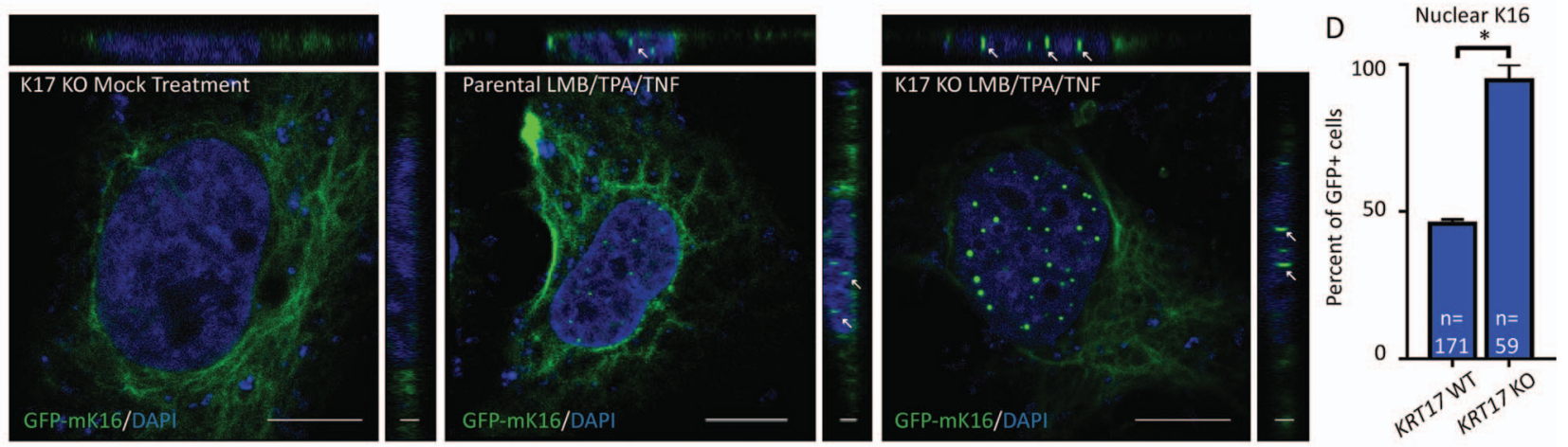

E
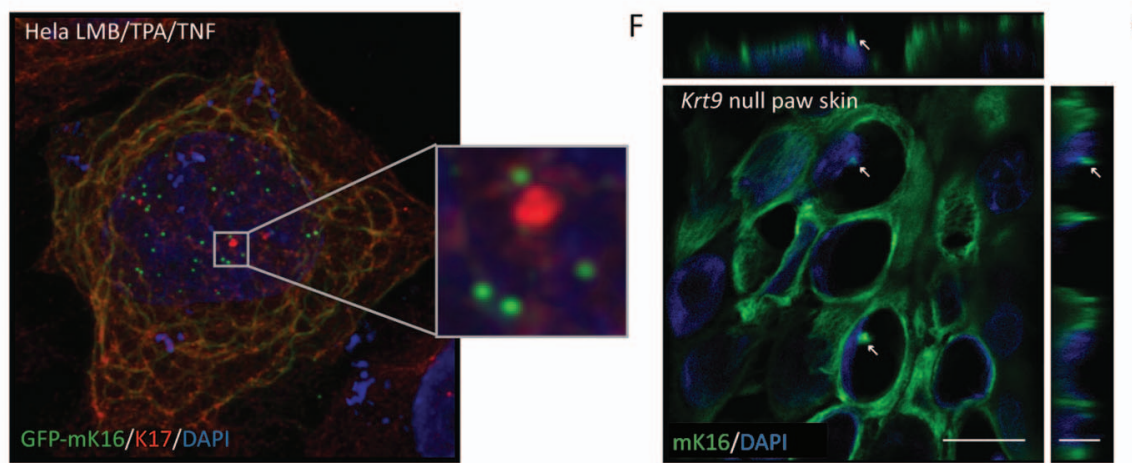

G

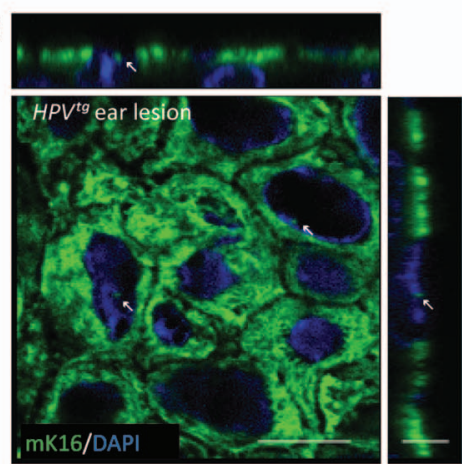

Figure 8. Nuclear localization of keratin 16. (A) Representative confocal images of HeLa and A431 cells transiently transfected with GFP-mK16 displaying nuclear K16 puncta after LMB/TPA/TNF treatment. Arrows mark examples of nuclear K16 puncta. Scale bars, $10 \mu \mathrm{m}$ (xy-plane) and $2 \mu \mathrm{m}$ (z-plane). (B) Confocal image of HeLa cells transiently transfected with human K16 and treated with LMB/TPA/TNF. Scale bars, $10 \mu \mathrm{m}$ (xy-plane) and $2 \mu \mathrm{m}$ (z-plane). (C) Representative confocal images of HeLa cells +/- K17 transiently transfected with GFP-mK16 and treated with either mock or LMB/TPA/TNF. Scale bars, 10 um (xy-plane) and 2 um (z-plane). Arrows mark examples of nuclear K16 puncta. (D) Quantitation of the percentage of transfected HeLa cells presenting with nuclear K16 signal from each genotype after LMB/TPA/TNF treatment. Number of cells counted per condition is denoted on the histogram. Error bars are SEM. (E) Confocal image of a HeLa cell transfected with GFP-mK16 and immunostained for K17 demonstrating that K16 and K17 do not co-localize when both are present in the nucleus. (F) Confocal image of immunostaining for endogenous $\mathrm{K} 16$ in paw skin tissue of 2 month old Krt9-null paw skin lesions. Arrows mark examples of nuclear K16 puncta. Scale bars, $10 \mu \mathrm{m}$ (xy-plane) and $5 \mu \mathrm{m}(\mathrm{z}$-plane). $\mathrm{N}=2$ mice. (G) Confocal image of immunostaining for endogenous $\mathrm{K} 16$ in murine ear tissue of a male HPV ${ }^{\mathrm{tg} /+}$ mouse at 4 months of age. Arrows mark examples of nuclear K16 puncta. Scale bars, $10 \mu \mathrm{m}$ (xy-plane) and $5 \mu \mathrm{m}$ (z-plane). $\mathrm{N}=2$ mice. All images are single confocal slices through the cell nucleus as determined by DAPI staining. $* P<0.05$.

skin within the same individual) (21). Interestingly, our analysis suggests a better correlation between Krt16-null mouse paw lesions and the data sets for individuals with missense alleles in KRT6. This likely reflects in part the limited number of human cases examined and the fact that the mouse model involves a null mutation whereas the human PC cases harbor dominantly acting missense alleles. It also is consistent with the complex phenotype-genotype correlations that typify PC.

Mutations in either KRT16 or KRT9 can cause PPK, though the associated presentations differ significantly. KRT16 mutations result in PPK lesions that are non-epidermolytic and focal, i.e. restricted to pressure points on the palmar and/or plan- tar epidermis. Mutations in KRT9 cause PPK lesions that are epidermolytic and diffuse, i.e. they occur throughout the palmar and/or plantar surface. The footpad skin lesions that spontaneously develop in Krt16-null mice resemble FNEPPK as they occur predominantly on pressure points with the substratum and show limited suprabasal cell lysis (10). As type I keratins, $\mathrm{K} 16$ and $\mathrm{K} 9$ would be expected to show some degree of functional redundancy in vivo $(62,63)$. Yet, the dramatic reduction of $\mathrm{Krt9/K9}$ levels in Krt16-null footpad skin is not associated with conversion of the PPK-like lesions from non-epidermolytic to epidermolytic. The low levels of K9 prevailing in Krt16-null footpad skin may suffice to confer mechanical resiliency to footpad 
skin epidermis, but it could also be that other factors are at play in the relationship between keratin proteins and/or filaments and mechanical resiliency per se in keratinocytes (64). Besides, the occurrence of a robust induction of Krt16/K16 in Krt9-null paw skin lesions (13) does not suffice to restore mechanical resiliency in this tissue. These findings attest to the multifaceted properties and sophisticated cellular roles of individual keratin proteins in skin.

Our efforts to directly implicate known effectors of terminal epidermal differentiation, including Nrf2, WNT, Notch, Dlx3, Hippo/Yap1 and AhR in the loss of Krt9/K9 expression at a prelesional stage in Krt16-null footpad skin have been unsuccessful. In nearly all cases, a survey of established target genes suggested that these pathways are, if anything, stimulated at 2 weeks of age in Krt16-null footpad skin, perhaps in an attempt to stimulate keratinocyte differentiation in response to a defective skin barrier (11). The lack of a direct role for Keap1-Nrf2 in regulating Krt9 expression was unanticipated given that the gene features several predicted AREs, and hypoactive Nrf2 plays a role at the time of lesion onset in Krt16-null footpad skin $(17,18)$. These observations establish that all of these signaling pathways do not effect a straightforward transcriptional activation of the Krt9 locus though, admittedly, they do not rule out alternative mechanisms of gene regulation. For instance, there is strong evidence for microRNA-based regulation of keratin gene transcripts in vivo, including MiR-184 targeting of KRT15 to promote keratinocyte differentiation (65), MiR-486-3p and MiR-138 targeting of KRT17 in psoriasis $(66,67)$ and MiR-26a targeting of KRT19 in cholangiocarcinoma cells (68). Various algorithms (e.g. TargetScan, MiRDB) predict that microRNAs MiR-671-5p, MiR-67215p, MiR-4768-3p, MiR-4786-3p, MiR-4455, MiR-9500, MiR-6079 and MiR-4690-5p target the human KRT9 transcript, whereas microRNAs MiR-669-3p, MiR-344i, MiR-8097 and MiR-3073a-3p target the mouse Krt9 transcript. Several microRNAs, albeit distinct ones (MiR-28c, MiR-1983, MiR-31, MiR-6384 and MiR-7649), are upregulated by $\geq 3$ SDs above WT in the Krt16-null microarray data set. Clearly, additional studies are needed to examine the mechanisms responsible for Krt9/KRT9 regulation in volar skin tissue in vivo.

Recently, a functional interaction between $\mathrm{K} 16$ and the inactive rhomboid protease RHBDF2 (irhom2) has been shown to occur in keratinocytes. Autosomal dominant gain of function mutations in RHBDF2 are the underlying cause of tylosis with oesophageal cancer (TOC, OMIM:148500), and these individuals present with PPK lesions early in childhood. In TOC-derived keratinocytes, K16 dissociates from its preferred type II keratin protein partner $\mathrm{K} 6$ and instead binds irhom 2 and migrates to the perinuclear compartment (69). Furthermore, in palmoplantar keratinocytes, irhom 2 is positively regulated by the transcription factor p63, which promotes the proliferation of basal keratinocytes and inhibits terminal differentiation. In TOC patientderived keratinocytes, p63 perpetuates irhom2 activity, which promotes inflammation, oxidative stress and inhibits apoptosis. Treatment with SF suppresses this newly defined p63-irhom2 axis (70). The role of this newly elucidated p63-irhom2 axis in KRT16 mutation-driven disease, such as PC-associated PPK, has yet to be understood and warrants investigation.

As noted in Cao et al. (21), KRT9 transcripts are decreased in individuals harboring KRT6A mutations (8-fold to 36-fold decrease) but are minimally changed in those with KRT16 mutations. Of note, the levels of KRT16 varied greatly between PC cases. In KRT6 cases, the diminished KRT9 levels were accompanied by a 2- or 4-fold increase in KRT16 transcript levels, whereas the KRT16 cases, which had a modest ( 2- fold) increase in KRT9 levels, exhibited a remarkable 25- or 40fold increase in KRT16 transcript levels. The latter raises the possibility that the dramatic upregulation of KRT16, either the mutant or WT allele, in the KRT16 cases may help mitigate the dramatic decrease in KRT9 levels that occurs in individuals with lower KRT16 transcript levels. Whether these observations imply that Krt16-null mice are a better model for KRT6 mutation-based PPK lesions, and/or suggest that KRT9 re-expression may be most effective in treating PC cases arising from KRT6 mutations, requires further study.

The observation that K16 can localize to the nucleus of keratinocytes raises the question of what function(s), if any, K16 carries out while present in the nucleus. One obvious possibility is that nuclear-localized K16 could play a role in regulating transcription either through interactions with transcriptional machinery, or via an impact on chromatin structure. We previously reported that the expression levels for many genes involved in epidermal homeostasis depend upon $\mathrm{K} 16$, but here again the mechanism(s) have not been elucidated (11). The exact mechanism of how K16 localizes to the nucleus and what function(s) it may carry out in this locale have not been elucidated but represent an exciting opportunity to explore new biology.

The data presented here indicate that $\mathrm{Krt16/ \textrm {K } 1 6}$ is a positive regulator of $\mathrm{Krt9} / \mathrm{K} 9$ expression, and that complete loss of Krt16/K16 impairs the differentiation and function of volar skin keratinocytes despite the upregulation of many effectors of epidermal differentiation. The extent to which these newly defined roles are cell-autonomous versus cell non-autonomous remains unclear at present. We propose that impaired terminal differentiation is an early driver of PPK lesion onset and progression, since it occurs ahead of oxidative stress (17), and that stimulation of KRT9 expression might be a therapeutic avenue worth exploring for at least some cases of PC-associated PPK. Keratin 9 expression can be stimulated in human keratinocyte cultures via treatment with the GSK inhibitor SB216763 (27) or in mouse paw skin tissue by SF (this study). While SB21673 has not been used in clinical trials, SF in the form of broccoli sprout extract can be safely delivered topically to modulate keratin expression and has shown therapeutic promise in the treatment of epidermis bullosa simplex arising from mutations in either keratins K5 or K14 (63,71). Future studies are necessary to determine whether the progress fostered by our study can translate into an impact for the treatment of PC-associated PPK.

\section{Materials and Methods}

\section{Mouse strains and antibodies}

Krt16-null mice (10) were generated by our laboratory in the C57BL/6 strain background, fed chow and water ad libitum and maintained under specific pathogen-free conditions. Nrf2-null mice (C57Bl/6 background) were provided by Thomas Kensler (Johns Hopkins University) and genotyped as described (63). IL$1 R^{-/-}$mice (C57Bl/ 6 background) were obtained from the Jackson Laboratory Bar Harbor, Maine, USA and genotyped as described (26). Krt9-null mice (C57Bl/6 background) were provided by Irwin McLean (University of Dundee) and genotyped as described (13). HPV16 $6^{\mathrm{t} /+}$ transgenic mice (FVB/N strain) (72) were obtained from the National Cancer Institute. Commercial antibodies used are listed in Supplementary Material, Table 9. All secondary antibodies used were either Alexa Fluor-conjugated (Life Technologies) or HRP-conjugated (Sigma-Aldrich). Generation of antihK16, anti-K17 and anti-mK16 antibodies have been described $(57,73,74)$. 


\section{Mouse Studies}

All mouse studies have been reviewed and approved by the Institutional Animal Use and Care Committee at both Johns Hopkins University and the University of Michigan. Four week old Krt16-null mice were topically treated with either $100 \mu \mathrm{l}$ of 1 $\mu$ mole SF or vehicle (jojoba oil) control on their front paws twice weekly for 4 weeks (17). Dilutions of SF were made fresh the day of treatment. At the end of the treatment regimen, paw tissue was taken for biochemical and morphological analyses. For embryonic tissues, matings were initiated and embryos timed as described (75).

\section{Cell culture and transfection assays}

Parental A431 and HeLa cells were obtained from AATC and KRT17 knock out cell lines, which were generated at our laboratory have been described (55). Plasmids GFP-mK16 and CMVhK16 were previously generated and used in our laboratory $(17,76)$. The keratin 16 constructs were transiently transfected into A431 or Hela cells using FuGene HD (Promega \#E2311) at a 1:3 ratio (DNA:FuGene volume ratio) according to manufacturer's instructions.

\section{Chemicals}

Leptomycin B (LMB; Sigma \#P1585) was dissolved in 70\% methanol and used at $40 \mathrm{~nm}$ working concentration. TPA (Sigma \#L2913) was dissolved in dimethyl sulfoxide and used at $200 \mathrm{nM}$ working concentration. TNF (Biolegend 570102) was dissolved in phosphate buffered saline (PBS) and used at $100 \mathrm{~nm}$ working concentration. All drug treatments lasted for $3 \mathrm{~h}$ with a $30 \mathrm{~min}$ LMB pretreatment. SF was obtained from LKT Laboratories Inc.

\section{Microarray analysis}

At the time of harvest, forepaws were degloved to isolate paw skin, and the nonvolar skin was removed with a razor blade. Total RNA isolated from paw skin, epidermis and dermis, of 2 month old Krt16-null (2 males, 2 females) and WT ( 2 males, 2 females) littermates (see Biochemical and morphological analyses) was subjected to microarray analysis by the Johns Hopkins Deep Sequencing Microarray Core using the Affymetrix Mouse Transcriptome Array 1.0 GeneChip. Affymetrix CEL files were extracted with the Partek Genomics Suite 6.6 platform. Affymetrix junction probes were excluded. Data were quantile normalized to generate log2 transcript signal values that were used in subsequent ANOVA analysis. Pathway analysis was done with QIAGEN IPA software (build version $478438 \mathrm{M}$ ) on genes whose expression differed by 2 SDs or more in Krt16null relative to WT. The human PPK data were downloaded from the supplemental material of Cao et al. (21). The KRT6 and KRT16 data were selected and corresponding mouse orthologs were retrieved from Ensemble Biomart (77). The data were then merged with mouse Krt16 null data based on mouse Entrez ID. The full merged table is in Supplementary Material, Table 10. Pearson correlation analysis was performed using the fold change of each sample in the merged data. For gene overlap analysis, differentially expressed genes were selected applying the same criteria as in Cao et al. (21) for the KRT6 or KRT16 cases and applying a fold change $\geq 3$ SD for the mouse data (this study). The significance of the overlap is calculated by hypergeometric test.

\section{Biochemical and morphological analyses}

RNA was extracted from front paws using TRIzol reagent (Life Technologies) and treated with DNase (Nucleospin RNA kit; Machery Nagel). A total of $1 \mu \mathrm{g}$ of the total RNA from each sample was reverse transcribed (iScript cDNA synthesis kit; Bio-Rad), and RT-qPCR was performed as described using Actin and $18 \mathrm{~S}$ as reference genes $(17,55)$. The targetspecific oligonucleotide primer sets we used are listed in Supplementary Material, Table 11. Protein analysis was done by extracting protein from front paw skin tissue using TRIzol reagent (Life Technologies) according to the manufacturer's protocol and was solubilized in urea buffer [6.5 M urea, $50 \mathrm{~mm}$ Tris pH 7.5, $1 \mathrm{~mm}$ ethylene glycol tetraacetic acid, $2 \mathrm{~mm}$ dithiothreitol, $50 \mathrm{~mm}$ sodium fluoride, $1 \mathrm{~mm}$ sodium vanadate, $0.1 \%$ sodium dodecyl sulfate (SDS), sodium deoxycholate $0.01 \mathrm{~g} / \mathrm{ml}$ and protease inhibitors] overnight at $4^{\circ} \mathrm{C}$. The Bradford Assay Kit (Bio-Rad) was used to determine protein concentration, and equal amounts of protein (15-20 $\mu \mathrm{g}$ range) were loaded onto $12 \%$ SDS/PAGE gels and blotted onto nitrocellulose. Bound primary antibodies were detected with enhanced chemiluminescence (Thermo Scientific). Actin was used as a loading control in all immunoblots. Total glutathione, GSH and GSSG levels in paw tissue were measured using a Glutathione Fluorometric assay kit (BioVision) according to manufacturer's specifications. For histological analyses at 2 weeks of age, paw tissue samples were fixed in 4\% paraformaldehyde (Fisher Scientific \#50980487) overnight and embedded in paraffin the next day. Sectioning was done in a specific and consistent orientation relative to paw morphology, and sections stained with hematoxylin and eosin (H\&E) for routine histopathology. At harvest, paw tissue was alternatively submerged in optimal cutting temperature media (Sakura Finetek), flash frozen in liquid nitrogen and stored at $-40^{\circ} \mathrm{C}$ upon sectioning. Cryosections $(5-6 \mu \mathrm{m})$ were cut in a specific and consistent orientation relative to paw morphology and stained with H\&E for routine histopathology or incubated with primary antibodies and Alexa Fluor-conjugated secondary antibodies for indirect immunofluorescence $(17,55)$. Immunohistochemistry and immunofluorescence images were acquired using a Zeiss fluorescence microscope with Apotome attachment. Confocal immunofluorescence images were acquired using a Zeiss LSM 710 or LSM 800 microscope. The nuclear presence of K16 was assessed by single-plane confocal microscopy through the nucleus in preparations dual stained with DAPI, as described in (55). Images for the same marker were acquired at the same exposure, pixel range and gamma values. Acquired images were equally brightened, contrasted and cropped using ImageJ National Institutes of Health (NIH; version 1.51p) or Zen 2.3 software for optimal presentation. The PPK Index was determined as described in (17) by three blinded assessors working independently.

\section{Statistics and quantitation of images}

Unpaired two-tailed Student's $t$ tests were performed when appropriate. Significance was set at $P$-value less than 0.05 $(P<0.05)$. Quantification data were presented in mean + standard error of the mean (SEM) calculated by dividing the SD between biological replicates by the square root of the number of biological replicates. Epidermal thickness measurements were done by determining the average thickness of the full epidermis (three measurements/image) from four $10 \times$ Apotome images of each biological replicate using ImageJ software (NIH; version 1.50e). Basal cell aspect ratio was determined by diving the 
length and width of individual basal cell nuclei using the Image (NIH; version 1.50e) software. Quantitation of total protein levels from western blots and immunofluorescence images was also done using ImageJ software (NIH; version 1.50 e or 1.51p).

\section{Supplementary Material}

Supplementary Material is available at HMG online.

\section{Acknowledgements}

The authors are grateful to Luiz A. Garza (Johns Hopkins University) and Dongwon Kim (Johns Hopkins University) for insightful discussions and advice. We also thank Conover Talbot, Jr. (Johns Hopkins University Deep Sequencing Microarray Core) for assistance with microarray analysis, Deena Pedrioli (University of Zurich) for the generous gift of anti-mouse K9 antibody and members of the Coulombe laboratory, particularly Ryan Hobbs, Justin Jacob, Michelle Kerns and Joseph Shen for their support.

Conflict of Interest statement. None declared.

\section{Funding}

National Institute of Arthritis, Musculoskeletal and Skin Disease (grant AR044232 to P.A.C.); National Cancer Institute (grant T32 CA009110 to A.G.Z.).

\section{References}

1. Schiller, S., Seebode, C., Hennies, H.C., Giehl, K. and Emmert, S. (2014) Palmoplantar keratoderma (PPK): acquired and genetic causes of a not so rare disease. J. Dtsch. Dermatol. Ges., 12, 781-788.

2. Reis, A., Hennies, H.C., Langbein, L., Digweed, M., Mischke, D., Drechsler, M., Schrock, E., Royer-Pokora, B., Franke, W.W., Sperling, K. et al. (1994) Keratin 9 gene mutations in epidermolytic palmoplantar keratoderma (EPPK). Nat. Genet., 6, 174-179.

3. Arin, M.J., Longley, M.A., Kuster, W., Huber, M., Hohl, D., Rothnagel, J.A. and Roop, D.R. (1999) An asparagine to threonine substitution in the 1A domain of keratin 1: a novel mutation that causes epidermolytic hyperkeratosis. Exp. Dermatol., 8, 124-127.

4. Shamsher, M.K., Navsaria, H.A., Stevens, H.P., Ratnavel, R.C., Purkis, P.E., Kelsell, D.P., McLean, W.H., Cook, L.J., Griffiths, W.A., Gschmeissner, S. et al. (1995) Novel mutations in keratin 16 gene underly focal non-epidermolytic palmoplantar keratoderma (NEPPK) in two families. Hum. Mol. Genet., 4, 1875-1881.

5. Wilson, N.J., Messenger, A.G., Leachman, S.A., O'Toole, E.A., Lane, E.B., McLean, W.H. and Smith, F.J. (2010) Keratin K6c mutations cause focal palmoplantar keratoderma. J. Invest. Dermatol., 130, 425-429.

6. McLean, W.H., Rugg, E.L., Lunny, D.P., Morley, S.M., Lane, E.B., Swensson, O., Dopping-Hepenstal, P.J., Griffiths, W.A., Eady, R.A., Higgins, C. et al. (1995) Keratin 16 and keratin 17 mutations cause pachyonychia congenita. Nat. Genet., 9, 273-278.

7. Smith, F.J., Jonkman, M.F., van Goor, H., Coleman, C.M., Covello, S.P., Uitto, J. and McLean, W.H. (1998) A mutation in human keratin $\mathrm{K} 6 \mathrm{~b}$ produces a phenocopy of the $\mathrm{K} 17$ disorder pachyonychia congenita type 2. Hum. Mol. Genet., 7, 1143-1148.

8. Lin, M.T., Levy, M.L., Bowden, P.E., Magro, C., Baden, L., Baden, H.P. and Roop, D.R. (1999) Identification of sporadic mutations in the helix initiation motif of keratin 6 in two pachyonychia congenita patients: further evidence for a mutational hot spot. Exp. Dermatol., 8, 115-119.

9. Bowden, P.E., Haley, J.L., Kansky, A., Rothnagel, J.A., Jones, D.O. and Turner, R.J. (1995) Mutation of a type II keratin gene (K6a) in pachyonychia congenita. Nat. Genet., 10, 363-365.

10. Lessard, J.C. and Coulombe, P.A. (2012) Keratin 16-null mice develop palmoplantar keratoderma, a hallmark feature of pachyonychia congenita and related disorders. J. Invest. Dermatol., 132, 1384-1391.

11. Lessard, J.C., Pina-Paz, S., Rotty, J.D., Hickerson, R.P., Kaspar, R.L., Balmain, A. and Coulombe, P.A. (2013) Keratin 16 regulates innate immunity in response to epidermal barrier breach. Proc. Natl. Acad. Sci. U. S. A., 110, 19537-19542.

12. Zieman, A. and Coulombe, P.A. (2019) Pathophysiology of pachyonychia congenita-associated palmoplantar keratoderma: new insight into skin epithelial homeostasis. Br. J. Dermatol. In revision.

13. Fu, D.J., Thomson, C., Lunny, D.P., Dopping-Hepenstal, P.J., McGrath, J.A., Smith, F.J.D., Irwin McLean, W.H. and Leslie Pedrioli, D.M. (2014) Keratin 9 is required for the structural integrity and terminal differentiation of the palmoplantar epidermis. J. Invest. Dermatol., 134, 754-763.

14. Zieman, A. and Coulombe, P.A. (2018) The keratin 16 null phenotype is modestly impacted by genetic strain background in mice. Exp. Dermatol., 27, 672-674.

15. Fu, T., Leachman, S.A., Wilson, N.J., Smith, F.J., Schwartz, M.E. and Tang, J.Y. (2011) Genotype-phenotype correlations among pachyonychia congenita patients with K16 mutations. J. Invest. Dermatol., 131, 1025-1028.

16. Spaunhurst, K.M., Hogendorf, A.M., Smith, F.J., Lingala, B., Schwartz, M.E., Cywinska-Bernas, A., Zeman, K.J. and Tang, J.Y. (2012) Pachyonychia congenita patients with mutations in KRT6A have more extensive disease compared with patients who have mutations in KRT16. Br. J. Dermatol., 166, 875-878.

17. Kerns, M.L., Hakim, J.M., Lu, R.G., Guo, Y., Berroth, A., Kaspar, R.L. and Coulombe, P.A. (2016) Oxidative stress and dysfunctional NRF2 underlie pachyonychia congenita phenotypes. J. Clin. Invest., 126, 2356-2366.

18. Kerns, M.L., Hakim, J.M.C., Zieman, A., Lu, R.G. and Coulombe, P.A. (2018) Sexual dimorphism in response to an NRF2 inducer in a model for pachyonychia congenita. J. Invest. Dermatol., 138, 1094-1100.

19. Mi, H., Muruganujan, A. and Thomas, P.D. (2013) PANTHER in 2013: modeling the evolution of gene function, and other gene attributes, in the context of phylogenetic trees. Nucleic Acids Res., 41, D377-D386.

20. Thomas, P.D., Campbell, M.J., Kejariwal, A., Mi, H., Karlak, B., Daverman, R., Diemer, K., Muruganujan, A. and Narechania, A. (2003) PANTHER: a library of protein families and subfamilies indexed by function. Genome Res., 13, 2129-2141.

21. Cao, Y.A., Hickerson, R.P., Seegmiller, B.L., Grapov, D., Gross, M.M., Bessette, M.R., Phinney, B.S., Flores, M.A., Speaker, T.J., Vermeulen, A. et al. (2015) Gene expression profiling in pachyonychia congenita skin. J. Dermatol. Sci., 77, 156-165.

22. Fischer, H., Langbein, L., Reichelt, J., Buchberger, M., Tschachler, E. and Eckhart, L. (2016) Keratins K2 and 
$\mathrm{K} 10$ are essential for the epidermal integrity of plantar skin. J. Dermatol. Sci., 81, 10-16.

23. Sims, J.E. and Smith, D.E. (2010) The IL-1 family: regulators of immunity. Nat. Rev. Immunol., 10, 89-102.

24. Martinon, F., Mayor, A. and Tschopp, J. (2009) The inflammasomes: guardians of the body. Annu. Rev. Immunol., 27, 229-265.

25. Chong, H.C., Tan, M.J., Philippe, V., Tan, S.H., Tan, C.K., $\mathrm{Ku}$, C.W., Goh, Y.Y., Wahli, W., Michalik, L. and Tan, N.S. (2009) Regulation of epithelial-mesenchymal IL-1 signaling by PPARbeta/delta is essential for skin homeostasis and wound healing. J. Cell Biol., 184, 817-831.

26. Glaccum, M.B., Stocking, K.L., Charrier, K., Smith, J.L., Willis, C.R., Maliszewski, C., Livingston, D.J., Peschon, J.J. and Morrissey, P.J. (1997) Phenotypic and functional characterization of mice that lack the type I receptor for IL-1. J. Immunol., 159, 3364-3371.

27. Kim, D., Hossain, M.Z., Nieves, A., Gu, L., Ratliff, T.S., Mi Oh, S., Park, A., Han, S., Yang, N.B., Qi, J. et al. (2016) To control site-specific skin gene expression, autocrine mimics paracrine canonical Wnt signaling and is activated ectopically in skin disease. Amer. J. Path., 186, 1140-1150.

28. Moll, R., Franke, W.W., Volc-Platzer, B. and Krepler, R. (1982) Different keratin polypeptides in epidermis and other epithelia of human skin: a specific cytokeratin of molecular weight 46,000 in epithelia of the pilosebaceous tract and basal cell epitheliomas. J. Cell Biol., 95, 285-295.

29. Rice, R.H., Durbin-Johnson, B.P., Salemi, M., Schwartz, M.E., Rocke, D.M. and Phinney, B.S. (2017) Proteomic profiling of pachyonychia congenita plantar callus. J. Proteomics, 165, 132-137.

30. Schweizer, J., Bowden, P.E., Coulombe, P.A., Langbein, L., Lane, E.B., Magin, T.M., Maltais, L., Omary, M.B., Parry, D.A., Rogers, M.A. et al. (2006) New consensus nomenclature for mammalian keratins. J. Cell Biol., 174, 169-174.

31. Hesse, M., Zimek, A., Weber, K. and Magin, T.M. (2004) Comprehensive analysis of keratin gene clusters in humans and rodents. Eur. J. Cell Biol., 83, 19-26.

32. McGowan, K. and Coulombe, P.A. (1998) The wound repairassociated keratins 6,16 , and 17 . Insights into the role of intermediate filaments in specifying keratinocyte cytoarchitecture. Subcell. Biochem., 31, 173-204.

33. Wawersik, M., Paladini, R.D., Noensie, E. and Coulombe, P.A. (1997) A proline residue in the alpha-helical rod domain of type I keratin 16 destabilizes keratin heterotetramers. J. Biol. Chem., 272, 32557-32565.

34. Takahashi, K., Yan, B., Yamanishi, K., Imamura, S. and Coulombe, P.A. (1998) The two functional keratin 6 genes of mouse are differentially regulated and evolved independently from their human orthologs. Genomics, 53, 170-183.

35. Zhang, Y., Talalay, P., Cho, C.G. and Posner, G.H. (1992) A major inducer of anticarcinogenic protective enzymes from broccoli: isolation and elucidation of structure. Proc. Natl. Acad. Sci. U. S. A., 89, 2399-2403.

36. Wakabayashi, N., Skoko, J.J., Chartoumpekis, D.V., Kimura, S., Slocum, S.L., Noda, K., Palliyaguru, D.L., Fujimuro, M., Boley, P.A., Tanaka, Y. et al. (2014) Notch-Nrf2 axis: regulation of Nrf2 gene expression and cytoprotection by notch signaling. Mol. Cell. Biol., 34, 653-663.

37. Lee, Y., Shin, J.M., Jang, S., Choi, D.K., Seo, M.S., Kim, H.R., Sohn, K.C., Im, M., Seo, Y.J., Lee, J.H. et al. (2014) Role of nuclear factor E2-related factor 2 (Nrf2) in epidermal differentiation. Arch. Dermatol. Res., 306, 677-682.
38. Ishitsuka, Y., Huebner, A.J., Rice, R.H., Koch, P.J., Speransky, V.V., Steven, A.C. and Roop, D.R. (2016) Lce1 family members are Nrf2-target genes that are induced to compensate for the loss of loricrin. J. Invest. Dermatol., 136, 1656-1663.

39. Schafer, M., Farwanah, H., Willrodt, A.H., Huebner, A.J., Sandhoff, K., Roop, D., Hohl, D., Bloch, W. and Werner, S. (2012) Nrf2 links epidermal barrier function with antioxidant defense. EMBO Mol. Med., 4, 364-379.

40. Endo, H., Sugioka, Y., Nakagi, Y., Saijo, Y. and Yoshida, T. (2008) A novel role of the NRF2 transcription factor in the regulation of arsenite-mediated keratin 16 gene expression in human keratinocytes. Environ. Health Perspect., 116, 873-879.

41. Itoh, K., Chiba, T., Takahashi, S., Ishii, T., Igarashi, K., Katoh, Y., Oyake, T., Hayashi, N., Satoh, K., Hatayama, I. et al. (1997) An Nrf2/small Maf heterodimer mediates the induction of phase II detoxifying enzyme genes through antioxidant response elements. Biochem. Biophys. Res. Commun., 236, 313-322.

42. Yamaguchi, Y., Itami, S., Tarutani, M., Hosokawa, K., Miura, H. and Yoshikawa, K. (1999) Regulation of keratin 9 in nonpalmoplantar keratinocytes by palmoplantar fibroblasts through epithelial-mesenchymal interactions. J. Invest. Dermatol., 112, 483-488.

43. Rinn, J.L., Wang, J.K., Allen, N., Brugmann, S.A., Mikels, A.J., Liu, H., Ridky, T.W., Stadler, H.S., Nusse, R., Helms, J.A. et al. (2008) A dermal HOX transcriptional program regulates sitespecific epidermal fate. Genes Deu., 22, 303-307.

44. Yamaguchi, Y., Itami, S., Watabe, H., Yasumoto, K., AbdelMalek, Z.A., Kubo, T., Rouzaud, F., Tanemura, A., Yoshikawa, K. and Hearing, V.J. (2004) Mesenchymal-epithelial interactions in the skin: increased expression of dickkopf1 by palmoplantar fibroblasts inhibits melanocyte growth and differentiation. J. Cell Biol., 165, 275-285.

45. Yamaguchi, Y., Passeron, T., Hoashi, T., Watabe, H., Rouzaud, F., Yasumoto, K., Hara, T., Tohyama, C., Katayama, I., Miki, T. et al. (2008) Dickkopf 1 (DKK1) regulates skin pigmentation and thickness by affecting Wnt/beta-catenin signaling in keratinocytes. FASEB J., 22, 1009-1020.

46. Rangarajan, A., Talora, C., Okuyama, R., Nicolas, M., Mammucari, C., Oh, H., Aster, J.C., Krishna, S., Metzger, D., Chambon, P. et al. (2001) Notch signaling is a direct determinant of keratinocyte growth arrest and entry into differentiation. EMBO J., 20, 3427-3436.

47. Hwang, J., Kita, R., Kwon, H.S., Choi, E.H., Lee, S.H., Udey, M.C. and Morasso, M.I. (2011) Epidermal ablation of Dlx3 is linked to IL-17-associated skin inflammation. Proc. Natl. Acad. Sci. U. S. A., 108, 11566-11571.

48. Miroshnikova, Y.A., Le, H.Q., Schneider, D., Thalheim, T., Rubsam, M., Bremicker, N., Polleux, J., Kamprad, N., Tarantola, M., Wang, I. et al. (2018) Adhesion forces and cortical tension couple cell proliferation and differentiation to drive epidermal stratification. Nat. Cell Biol., 20, 69-80.

49. Gormar, F.E., Bernd, A., Bereiter-Hahn, J. and Holzmann, H. (1990) A new model of epidermal differentiation: induction by mechanical stimulation. Arch. Dermatol. Res., 282, 22-32.

50. Yano, S., Komine, M., Fujimoto, M., Okochi, H. and Tamaki, K. (2004) Mechanical stretching in vitro regulates signal transduction pathways and cellular proliferation in human epidermal keratinocytes. J. Invest. Dermatol., 122, 783-790.

51. Totaro, A., Castellan, M., Battilana, G., Zanconato, F., Azzolin, L., Giulitti, S., Cordenonsi, M. and Piccolo, S. (2017) YAP/TAZ link cell mechanics to Notch signalling to control epidermal stem cell fate. Nat. Commun., 8, 15206. 
52. Schlegelmilch, K., Mohseni, M., Kirak, O., Pruszak, J., Rodriguez, J.R., Zhou, D., Kreger, B.T., Vasioukhin, V., Avruch, J., Brummelkamp, T.R. et al. (2011) Yap1 acts downstream of alpha-catenin to control epidermal proliferation. Cell, 144, 782-795.

53. Haas, K., Weighardt, H., Deenen, R., Kohrer, K., Clausen, B., Zahner, S., Boukamp, P., Bloch, W., Krutmann, J. and Esser, C. (2016) Aryl hydrocarbon receptor in keratinocytes is essential for murine skin barrier integrity. J. Invest. Dermatol., 136, 2260-2269.

54. van den Bogaard, E.H., Podolsky, M.A., Smits, J.P., Cui, X., John, C., Gowda, K., Desai, D., Amin, S.G., Schalkwijk, J., Perdew, G.H. et al. (2015) Genetic and pharmacological analysis identifies a physiological role for the AHR in epidermal differentiation. J. Invest. Dermatol., 135, 1320-1328.

55. Hobbs, R.P., DePianto, D.J., Jacob, J.T., Han, M.C., Chung, B.M., Batazzi, A.S., Poll, B.G., Guo, Y., Han, J., Ong, S. et al. (2015) Keratin-dependent regulation of AIRE and gene expression in skin tumor keratinocytes. Nat. Genet., 47, 933-938.

56. Hobbs, R.P., Jacob, J.T. and Coulombe, P.A. (2016) Keratins are going nuclear. Dev. Cell, 38, 227-233.

57. Takahashi, K., Folmer, J. and Coulombe, P.A. (1994) Increased expression of keratin 16 causes anomalies in cytoarchitecture and keratinization in transgenic mouse skin. J. Cell Biol., $127,505-520$.

58. Blessing, M., Ruther, U. and Franke, W.W. (1993) Ectopic synthesis of epidermal cytokeratins in pancreatic islet cells of transgenic mice interferes with cytoskeletal order and insulin production. J. Cell Biol., 120, 743-755.

59. Escobar-Hoyos, L.F., Shah, R., Roa-Pena, L., Vanner, E.A., Najafian, N., Banach, A., Nielsen, E., Al-Khalil, R., Akalin, A., Talmage, D. et al. (2015) Keratin-17 promotes p27KIP1 nuclear export and degradation and offers potential prognostic utility. Cancer Res., 75, 3650-3662.

60. Kumeta, M., Hirai, Y., Yoshimura, S.H., Horigome, T. and Takeyasu, K. (2013) Antibody-based analysis reveals 'filamentous vs. non-filamentous' and 'cytoplasmic vs. nuclear' crosstalk of cytoskeletal proteins. Exp. Cell Res., 319, 3226-3237.

61. Hobbs, R.P., Batazzi, A.S., Han, M.C. and Coulombe, P.A. (2016) Loss of keratin 17 induces tissue-specific cytokine polarization and cellular differentiation in HPV16-driven cervical tumorigenesis in vivo. Oncogene, 35, 5653-5662.

62. Wong, P., Domergue, R. and Coulombe, P.A. (2005) Overcoming functional redundancy to elicit pachyonychia congenitalike nail lesions in transgenic mice. Mol. Cell. Biol., 25, 197-205.

63. Kerns, M.L., DePianto, D., Dinkova-Kostova, A.T., Talalay, P. and Coulombe, P.A. (2007) Reprogramming of keratin biosynthesis by sulforaphane restores skin integrity in epidermolysis bullosa simplex. Proc. Natl. Acad. Sci. U. S. A., 104, 14460-14465.

64. Hatzfeld, M., Keil, R. and Magin, T.M. (2017) Desmosomes and intermediate filaments: their consequences for tissue mechanics. Cold Spring Harb. Perspect. Biol., 9:a029157.
65. Nagosa, S., Leesch, F., Putin, D., Bhattacharya, S., Altshuler, A., Serror, L., Amitai-Lange, A., Nasser, W., Aberdam, E., Rouleau, M. et al. (2017) microRNA-184 induces a commitment switch to epidermal differentiation. Stem Cell Reports, 9, 1991-2004.

66. Jiang, M., Sun, Z., Dang, E., Li, B., Fang, H., Li, J., Gao, L., Zhang, K. and Wang, G. (2017) TGFbeta/SMAD/microRNA486-3p signaling axis mediates keratin 17 expression and keratinocyte hyperproliferation in psoriasis. J. Invest. Dermatol., 137, 2177-2186.

67. Feng, S.J., Chu, R.Q., Ma, J., Wang, Z.X., Zhang, G.J., Yang, X.F., Song, Z. and Ma, Y.Y. (2017) MicroRNA138 regulates keratin 17 protein expression to affect HaCaT cell proliferation and apoptosis by targeting hTERT in psoriasis vulgaris. Biomed. Pharmacother., 85, 169-176.

68. Wang, P. and Lv, L. (2016) miR-26a induced the suppression of tumor growth of cholangiocarcinoma via KRT19 approach. Oncotarget, 7, 81367-81376.

69. Maruthappu, T., Chikh, A., Fell, B., Delaney, P.J., Brooke, M.A., Levet, C., Moncada-Pazos, A., Ishida-Yamamoto, A., Blaydon, D., Waseem, A. et al. (2017) Rhomboid family member 2 regulates cytoskeletal stress-associated Keratin 16. Nat. Commun., 8,14174 .

70. Arcidiacono, P., Webb, C.M., Brooke, M.A., Zhou, H., Delaney, P.J., Ng, K.E., Blaydon, D.C., Tinker, A., Kelsell, D.P. and Chikh, A. (2018) p63 is a key regulator of iRHOM2 signalling in the keratinocyte stress response. Nat. Commun., 9, 1021.

71. Kerns, M.L., Guss, L., Fahey, J., Cohen, B., Hakim, J.M., Sung, S., Lu, R.G. and Coulombe, P.A. (2017) Randomized, splitbody, single-blinded clinical trial of topical broccoli sprout extract: Assessing the feasibility of its use in keratin-based disorders. J. Amer. Acad. Dermatol., 76, 449-453.e441.

72. Arbeit, J.M., Howley, P.M. and Hanahan, D. (1996) Chronic estrogen-induced cervical and vaginal squamous carcinogenesis in human papillomavirus type 16 transgenic mice. Proc. Natl. Acad. Sci. U. S. A., 93, 2930-2935.

73. McGowan, K.M. and Coulombe, P.A. (1998) Onset of keratin 17 expression coincides with the definition of major epithelial lineages during skin development. J. Cell Biol., 143, 469-486.

74. Bernot, K.M., Coulombe, P.A. and McGowan, K.M. (2002) Keratin 16 expression defines a subset of epithelial cells during skin morphogenesis and the hair cycle. J. Invest. Dermatol., 119, 1137-1149.

75. Findlater, G.S., McDougall, R.D. and Kaufman, M.H. (1993) Eyelid development, fusion and subsequent reopening in the mouse. J. Anat., 183, 121-129.

76. Bernot, K.M., Lee, C.H. and Coulombe, P.A. (2005) A small surface hydrophobic stripe in the coiled-coil domain of type I keratins mediates tetramer stability. J. Cell Biol., 168, 965-974.

77. Kinsella, R.J., Kahari, A., Haider, S., Zamora, J., Proctor, G., Spudich, G., Almeida-King, J., Staines, D., Derwent, P., Kerhornou, A. et al. (2011) Ensembl BioMarts: a hub for data retrieval across taxonomic space. Database, 2011. doi: https://doi.org/10.1093/database/bar030. 